ARTICLE

\title{
Quantitative modeling predicts mechanistic links between pre-treatment microbiome composition and metronidazole efficacy in bacterial vaginosis
}

Christina Y. Lee (1) 1,8, Ryan K. Cheu ${ }^{2,3,8}$, Melissa M. Lemke (1) ${ }^{1}$, Andrew T. Gustin (1) 2,3, Michael T. France ${ }^{4}$, Benjamin Hampel ${ }^{5}$, Andrea R. Thurman ${ }^{6}$, Gustavo F. Doncel ${ }^{6}$, Jacques Ravel (1) ${ }^{4}$, Nichole R. Klatt (1) ${ }^{2,3,7,9 凶} \&$ Kelly B. Arnold (1D) 1,9凶

Bacterial vaginosis is a condition associated with adverse reproductive outcomes and characterized by a shift from a Lactobacillus-dominant vaginal microbiota to a polymicrobial microbiota, consistently colonized by strains of Gardnerella vaginalis. Metronidazole is the first-line treatment; however, treatment failure and recurrence rates remain high. To understand complex interactions between Gardnerella vaginalis and Lactobacillus involved in efficacy, here we develop an ordinary differential equation model that predicts bacterial growth as a function of metronidazole uptake, sensitivity, and metabolism. The model shows that a critical factor in efficacy is Lactobacillus sequestration of metronidazole, and efficacy decreases when the relative abundance of Lactobacillus is higher pre-treatment. We validate results in Gardnerella and Lactobacillus co-cultures, and in two clinical cohorts, finding women with recurrence have significantly higher pre-treatment levels of Lactobacillus relative to bacterial vaginosis-associated bacteria. Overall results provide mechanistic insight into how personalized differences in microbial communities influence vaginal antibiotic efficacy.

\footnotetext{
${ }^{1}$ Department of Biomedical Engineering, University of Michigan, Ann Arbor, MI, USA. ${ }^{2}$ University of Miami Department of Pediatrics, University of Miami, Miami, FL, USA. ${ }^{3}$ Department of Pharmaceutics, University of Washington, Seattle, WA, USA. ${ }^{4}$ Institute for Genome Sciences and Department of Microbiology and Immunology, University of Maryland School of Medicine, Baltimore, MD, USA. ${ }^{5}$ Division of Infectious Diseases and Hospital Epidemiology, University of Zurich, Zürich, Switzerland. ${ }^{6}$ CONRAD, Eastern Virginia Medical School, Norfolk, VA, USA. ${ }^{7}$ Department of Surgery, University of Minnesota, Minneapolis, MN, USA. ${ }^{8}$ These authors contributed equally: Christina Y. Lee, Ryan K. Cheu. ${ }^{9}$ These authors jointly supervised this work: Nichole R. Klatt, Kelly B. Arnold. ${ }^{凶}$ email: klat0037@umn.edu; kbarnold@umich.edu
} 
B acterial vaginosis (BV) is a condition that affects $30-60 \%$ of women worldwide ${ }^{1,2}$, with negative outcomes including increased susceptibility to sexually transmitted infections (STIs) and greater likelihood for adverse reproductive outcomes ${ }^{3-7}$. $\mathrm{BV}$ is characterized by a shift from Lactobacillus species (spp.)dominated vaginal microbiota to a diverse array of anaerobic bacteria including Gardnerella vaginalis $(G v)$ and Atopobium vaginae $^{8-10}$. Treatment of symptomatic BV with metronidazole (MNZ) aims to restore Lactobacillus-dominated microbiota; however, recurrence rates remain high, occurring in $57-90 \%$ of women who receive treatment ${ }^{11-14}$. Recurrence is associated with several host factors including previous episodes of BV, douching, and sexual activity, but no one factor emerges as a single driver of treatment failure ${ }^{11,15-18}$. In addition, associations between vaginal microbiota composition and BV recurrence have been reported but remain poorly understood, with several studies citing conflicting results ${ }^{15,17,19}$.

Recent improvements in 16S rRNA sequencing have enhanced the ability to identify and more accurately quantify the composition of the vaginal microbiota in $\mathrm{BV}^{20,21}$, finding that the transition is frequently associated with an abundance of Lactobacillus iners $(\mathrm{Li})^{22,23}$. Despite the association between $L i$ and incidence of $\mathrm{BV}$, identifying how $\mathrm{Li}$ dictates communities of optimal and non-optimal microbiota remains elusive, as the vaginal microbiota can change significantly over time and vary between women ${ }^{24-26}$, especially in the presence of MNZ. The recommended treatment regimen for $\mathrm{BV}$ consists of oral or vaginal MNZ oriented toward selectively targeting anaerobic bacteria with little effect on Lactobacillus spp. ${ }^{27,28}$, but high variability in efficacy indicates that further study is required to understand the reestablishment of optimal vaginal microbiota ecosystems.

Recent research in the HIV microbicide field has highlighted the importance of vaginal microbiome composition in drug treatment efficacy. In a landmark study, variability in tenofovir (TFV) microbicide efficacy was accounted for by differences in the vaginal microbiome, specifically the presence of the nontarget species $G v$, which were shown to metabolize $\mathrm{TFV}^{29}$. Likewise for MNZ treatment of Trichomonas vaginalis, a proposed mechanism of treatment failure was decreased bioavailability of MNZ due to the absorbance of the antibiotic by other microorganisms in the vagina ${ }^{30-32}$. In the context of $\mathrm{BV}$, it is difficult to discern the role of multiple possible interactions that have the potential to influence MNZ efficacy, including MNZ metabolism, resistance, and sequestration across multiple bacterial species that vary considerably among women. We propose that variability in MNZ efficacy may result from underlying differences in MNZ uptake and susceptibility in target and nontarget species, and therefore would be highly dependent on individual differences in pre-treatment vaginal microbiota composition.

In this work, we use an ordinary differential equation-based (ODE) model and experimentally measure parameters (MNZ internalization by bacteria, metabolism, and bacterial antibiotic susceptibility) to predict $L i$ and $G v$ growth dynamics with MNZ treatment. The model demonstrates that a critical factor in MNZ efficacy may be $L i$ sequestration of MNZ, and predicts that MNZ efficacy decreases in individuals with higher pre-treatment amounts of the non-target species, $L i$, relative to the target species, $G v$. We validate this finding with in vitro co-cultures, and extend our analysis to more representative models which illustrate that this behavior is also expected in microbial environments with additional species, interspecies interactions, and strain variability. Finally, by analyzing cervicovaginal samples from BV-infected women treated with MNZ in two distinct cohorts we demonstrate that our initial findings have clinical relevance in characterizing BV treatment outcomes ${ }^{33,34}$. Overall, our findings highlight the importance of leveraging quantitative models that evaluate interactions of target bacteria and non-target Lactobacillus spp. with MNZ in improving insight into personalized differences in $\mathrm{BV}$ recurrence and treatment failure.

\section{Results}

Model predicts Lactobacillus MNZ sequestration influences efficacy. To determine how MNZ treatment efficacy can be altered by bacterial-mediated interactions in vitro, we created an ODE model to predict growth of $G v$ and $L i$ upon co-culture and treatment with MNZ (Fig. 1a). Parameters for each bacterial species were obtained by least-squares fitting of in vitro kinetic data and dose-response curves for MNZ exposure with each species in monoculture (Supplementary Figs. 1, 2, Supplementary Table 1), before the ODE model was used to predict co-culture conditions with $G v$ and $L i$ both interacting with extracellular MNZ. The model assumes that $G v$ and $L i$ internalize or sequester MNZ at rates $k_{\text {int-Gv }}$ and $k_{\text {int-Li }}$, respectively, and $G v$ can convert MNZ to the stable metabolite, acetamide, and unknown metabolites at rate $k_{\text {met }}{ }^{35}$. The model additionally assumes logistic growth at rates $k_{\text {grow-Gv }}$ and $k_{\text {grow-Li }}$, with carrying capacities of $K_{\mathrm{Gv}}$ and $K_{\mathrm{Li}}$ and growth inhibition by MNZ toxicity at rates $k_{\text {kill- }}$ $\mathrm{Gv}$ and $k_{\mathrm{kill}-\mathrm{Li}}$ in a dose-dependent manner based on $50 \%$ effective concentrations of $\mathrm{MNZ}$ on $\mathrm{G} v$ and $\mathrm{Li}\left(\mathrm{EC} 50_{\mathrm{Gv}}, \mathrm{EC} 50_{\mathrm{Li}}\right)^{36,37}$. Since MNZ is a pro-drug that is activated when internalized by anaerobic bacteria, the cytotoxicity of MNZ in the model is dependent on the intracellular concentration of $\mathrm{MNZ}$ rather than extracellular MNZ concentration; however, we used the external MNZ concentration as the basis for EC50 of internalized MNZ, as experimentally determining the intracellular level of MNZ per cell was challenging and the main goal was to capture the relative sensitivity between $G v$ and $L i^{27,28,30,38}$.

To identify model parameters that were most critical for decreasing $G v$ growth, we performed a 1-dimensional (1D) sensitivity analysis by altering each parameter three orders of magnitude above and below baseline and evaluated $G v$ growth (Fig. 1b, c). Growth was scaled relative to the predicted growth in an unperturbed (no MNZ) co-culture based on the time point and initial population sizes evaluated and is referred to as percent maximum growth. The sensitivity analysis identified $G v$ growth as highly dependent on the MNZ internalization/sequestration rate into $L i\left(k_{\text {int-Li }}\right)$. A 50 -fold increase in this rate increased the growth of $G v$ from 7.42 to $69.5 \%$ its maximal growth upon $48 \mathrm{~h}$ treatment with MNZ (Fig. 1c). Likewise, changing the MNZ internalization rate into $G v\left(k_{\text {int-Gv }}\right)$ has similar effects on $L i$ where increasing this rate 50 -fold resulted in $89.7 \% \mathrm{Li}$ 's maximal growth (Supplementary Fig. 3a). Overall, these results illustrate how MNZ efficacy in inhibiting $G v$ growth is influenced by the competition between each bacterium to internalize the drug.

From this result, we hypothesized that the relative quantity of cells internalizing MNZ (ratio of $G v$ and $L i$ ) could significantly influence growth of both strains. We tested this hypothesis in our computational framework by predicting $G v$ survival after varying the starting ratio of $G v$ to $L i$ (Gv: $\mathrm{Li}$ ratio) from $1000 \times$ fold to $0.001 \times$. Results indicated that altering the initial Gv:Li ratio influences the growth of both $G v$ and $L i$. Counterintuitively, $G v$ survival was high when $L i$ initially outnumbers $G v 1000 \times$ and $L i$ growth is optimal when $G v$ initially exceeds $L i 1000 \times$ (Fig. 1d). Stated differently, the model suggested that more $G v$ present at MNZ treatment initiation resulted in a better treatment outcome. The importance of MNZ internalization rate into $L i$ became more apparent as $L i$ became the predominating species, leading to increased growth of $G v$ (Fig. 1e). This result additionally supports 
a
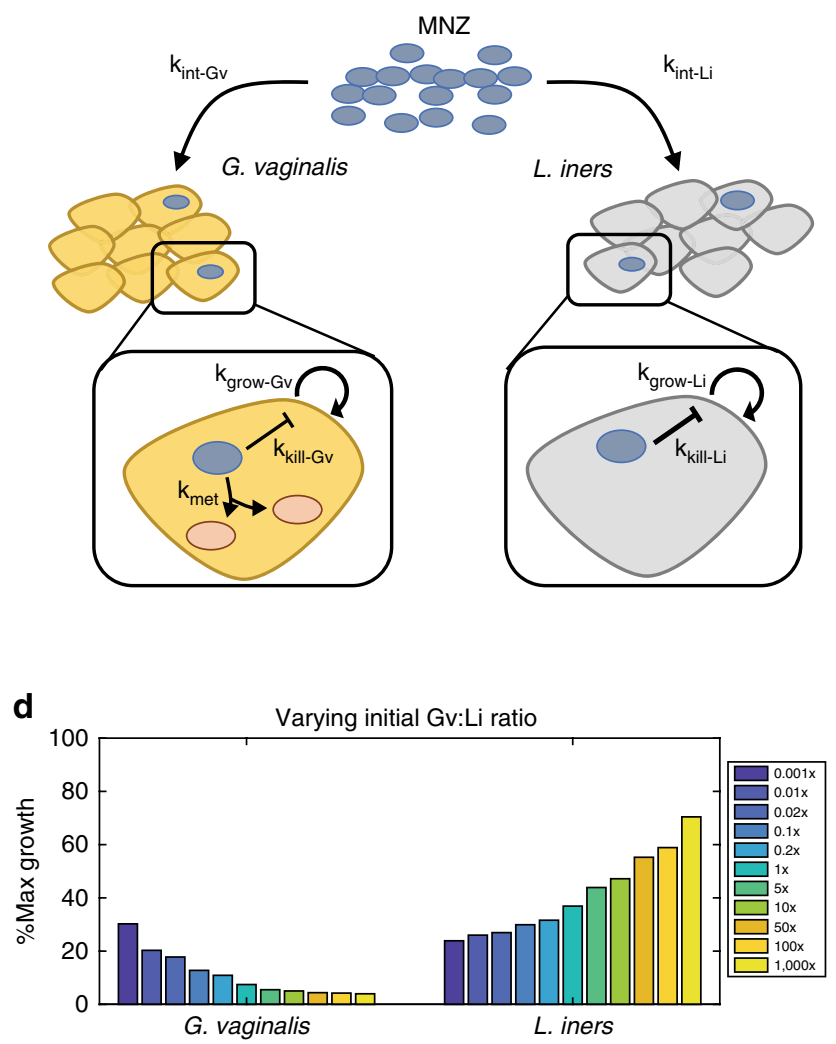

b
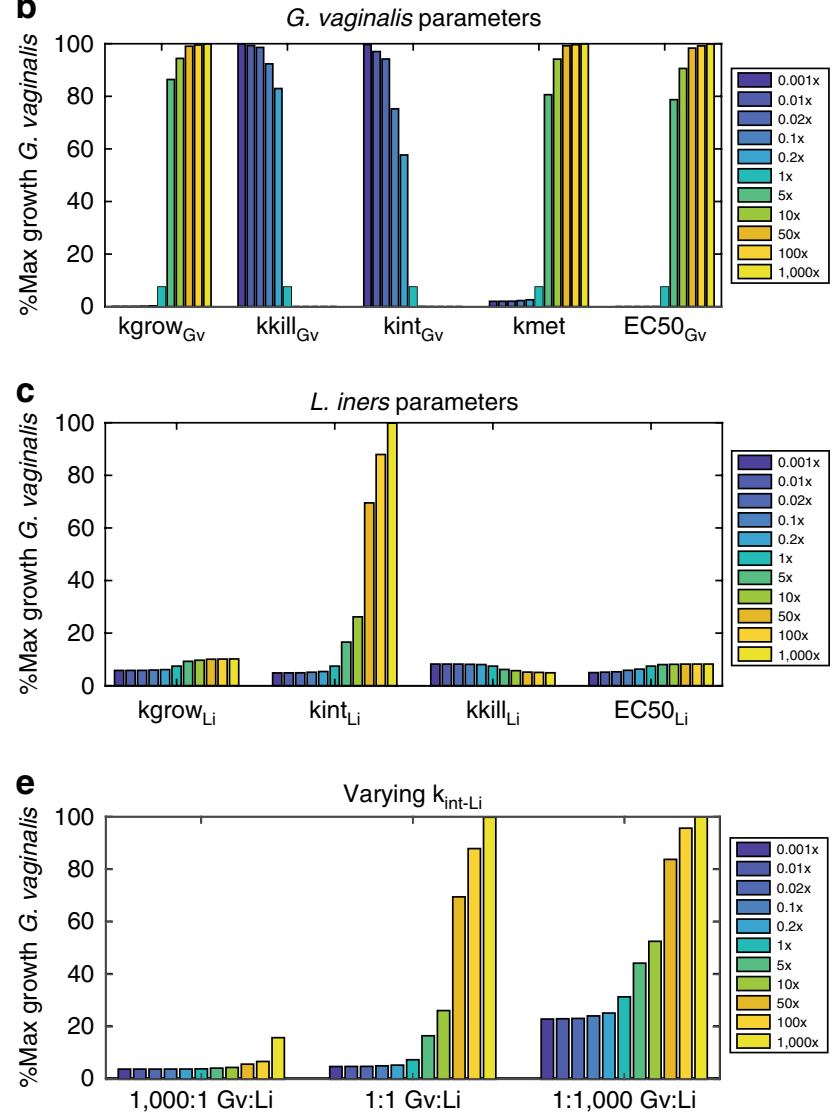

Fig. 1 Model schematic for bacterial growth dynamics in BV with MNZ treatment. a MNZ is internalized by both G. vaginalis ( $G v$ ) and $L$. iners ( $L i$ ) at rates $k_{\text {int-GV }}$ and $k_{\text {int-Ll, }}$ cells are proliferating at $k_{\text {grow-GV }}$ and $k_{\text {grow-Ll }}$ and MNZ inhibits growth by $k_{\text {kill-GV }}$ and $k_{\text {kill-LI. }}$. For $G V$, a potential mechanism of MNZ resistance is the bacterial-mediated interactions to the drug leading to the formation of metabolites $\left(k_{\mathrm{met}}\right)$. $\mathbf{b}$ Sensitivity of $\mathrm{Gv}$ growth with $500 \mu \mathrm{g} / \mathrm{ml} \mathrm{MNZ}$ when parameters directly related to Gv growth are varied $0.001 \times$ to $1000 \times$ baseline values. Percent maximal growth refers to the final cell count compared to the carrying capacity of the culture, or the maximum cell density the unperturbed culture can reach at $48 \mathrm{~h}$ based on initial cell density. $\mathbf{c}$ Sensitivity of Gv growth with $500 \mu \mathrm{g} / \mathrm{ml} \mathrm{MNZ}$ when parameters related to Li survival are varied 0.001× to 1000× baseline values. d Percent maximal growth of Gv (left) and $L i$ (right) when the initial ratio of $G v$ to $L i$ is varied with $500 \mu \mathrm{g} / \mathrm{ml}$ MNZ treatment. e Percent maximal growth of Gv when MNZ internalization rate of $L i$ is varied at three different population compositions with $500 \mu \mathrm{g} / \mathrm{ml}$ MNZ treatment.

the concept that $L i$ competes with $G v$ to internalize or sequester extracellular MNZ, as when one bacterial strain is in excess, it likely depletes available extracellular MNZ and decreases the amount of drug internalized by the non-dominating bacterial strain.

We used our model to explore this ratio-dependent behavior over a range of relevant MNZ concentrations extending from 100 to $1600 \mu \mathrm{g} / \mathrm{ml}$, as estimates for vaginal accumulation range from 20 to $>1000 \mu \mathrm{g} / \mathrm{ml}$ (Fig. 2a, b) ${ }^{39,40}$. Doses below $100 \mu \mathrm{g} / \mathrm{ml}$ had no effect on $G v$ or $L i$ growth and doses above $1600 \mu \mathrm{g} / \mathrm{ml}$ exhibited near-complete cell killing for both bacterial strains (Fig. 2a, b); these data are in agreement with experimentally determined effective concentrations of MNZ on $G v$ and $L i$ cultured individually (Supplementary Fig. 2c, d). However, for doses between 100 and $1600 \mu \mathrm{g} / \mathrm{ml}$ there were significant differences depending on the initial Gv:Li ratio, where $\mathrm{MNZ}$ was most efficacious in eliminating $G v$ when more $G v$ than $L i$ was present initially.

Model validation in $\boldsymbol{G} \boldsymbol{v}$ and $\boldsymbol{L i}$ co-cultures. We validated these counterintuitive model predictions experimentally in vitro by varying the initial Gv:Li ratios in the presence of $500 \mu \mathrm{g} / \mathrm{ml} \mathrm{MNZ}$ and tracking growth for $48 \mathrm{~h}$ (Fig. 2c, d). Experimental measurements confirmed model predictions that MNZ efficacy for inhibiting $G v$ growth decreased when $L i$ was initially dominant $\left(p=4.67 \times 10^{-8}\right)$, and were not significantly different than model predictions $(0.001 \times \mathrm{Gv}: \mathrm{Li}, p=0.430 ; 1000 \times \mathrm{Gv}: \mathrm{Li}$ ratio, $p=$ 0.689 , Fig. 3c), with $G v$ exhibiting a predicted $30.3 \%$ and experimental $41.4 \% \pm 13.3 \%$ maximal growth after treatment when $L i$ was initially dominant compared to a predicted $2.1 \%$ and experimental $9.4 \% \pm 13.8 \%$ maximal growth when $G v$ initially was dominant. $L i$ growth in the presence of $500 \mu \mathrm{g} / \mathrm{ml} \mathrm{MNZ} \mathrm{was}$ also dependent on the initial Gv:Li ratio, where MNZ inhibited $L i$ growth the most when $L i$ was initially dominant, $7.2 \% \pm 3.9 \%$ maximal growth compared to when $G v$ was initially dominant, $70.5 \% \pm 33.8 \%\left(p=3.29 \times 10^{-9}\right.$, Fig. $\left.2 \mathrm{~d}\right)$. Notably, the model over-predicted the growth of the $L i$ population when $L i$ was initially dominant $(0.001 \times \mathrm{Gv}: \mathrm{Li})$, where the model prediction of $23.9 \%$ maximal growth was over threefold higher than experimentally observed, $7.19 \% \pm 3.91 \%$ growth $(0.001 \times \mathrm{Gv}$ :Li experiment vs simulation, $\left.p=1.43 \times 10^{-5}\right)$, suggesting efficacy dependence on a high pre-treatment Gv:Li ratio may be even greater than that predicted by the model. Experimental and model predictions of $L i$ growth were not significantly different when $G v$ was initially dominant $(1000 \times \mathrm{Gv}: \mathrm{Li}, p=0.726)$. 
a

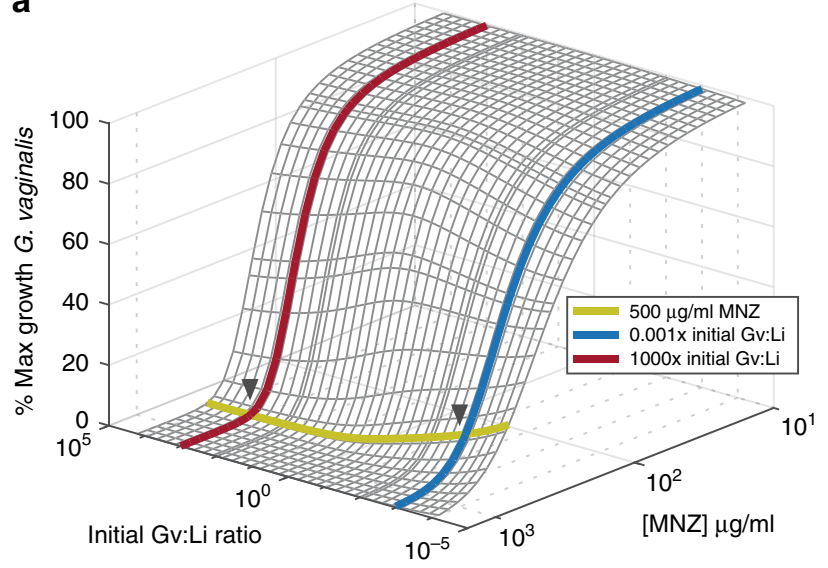

C

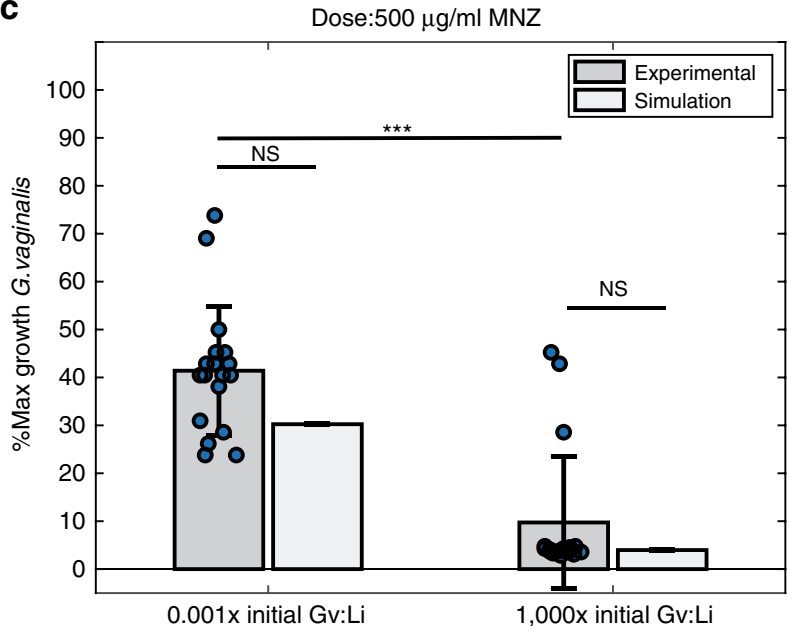

b

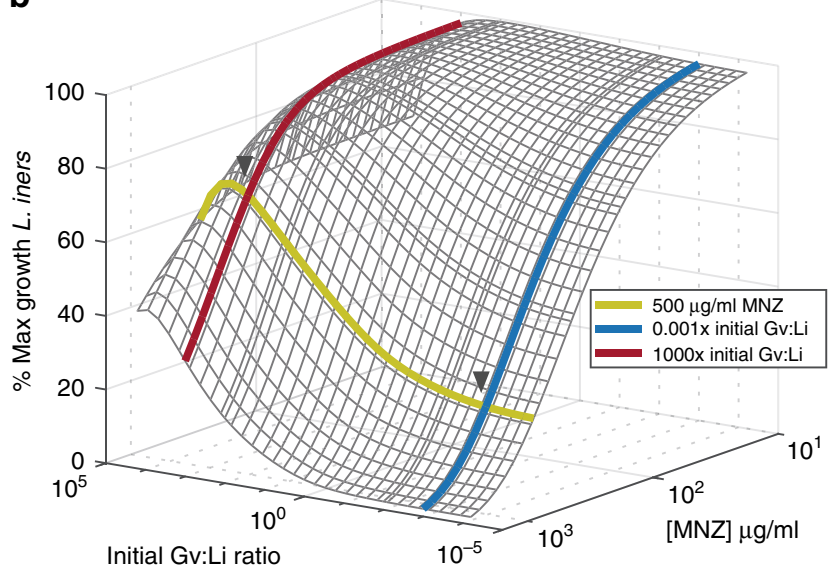

d

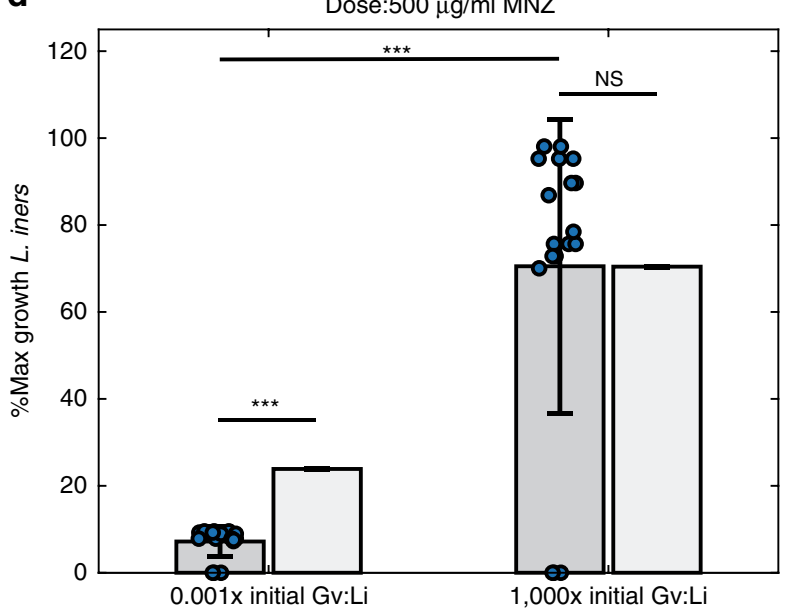

Fig. 2 A higher initial Gv:Li ratio improves MNZ treatment efficacy. a Surface plot to illustrate predicted percent maximal growth of Gv ( $z$-axis) when concentration of MNZ ( $x$-axis) and the ratio of Gv:Li ( $y$-axis) are varied in simultaneously. Arrows indicate the concentration of MNZ and ratios of Gv:Li used for model validation. $\mathbf{b}$ Percent maximal growth of $L i$ after simultaneous variation of MNZ dose and Gv:Li ratio. c, $\mathbf{d}$ Comparison of model simulations

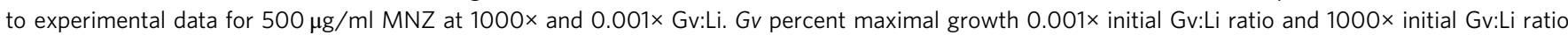
experimental vs simulation, and experimental vs experimental $p$-values were $p=0.430, t=0.809, \mathrm{df}=17 ; p=0.680, t=0.420, \mathrm{df}=17 ; p=4.67 \times 10^{-8}$, $t=6.99, \mathrm{df}=34$, respectively. Li percent maximal growth $0.001 \times$ initial Gv:Li ratio and 1000x initial Gv:Li ratio experimental vs simulation, and experimental vs experimental $p$-values were $p=1.43 \times 10^{-5}, t=6.00, \mathrm{df}=17 ; p=0.726, t=0.357, \mathrm{df}=17 ; p=3.29 \times 10^{-9}, t=7.91, \mathrm{df}=34, \mathrm{respectively}$. Data are presented as mean $\pm \mathrm{SD}, n=18$ independent, biological replicates for each initial ratio, asterisks indicate significance as: ${ }^{\star} p<0.05$, ${ }^{\star \star} p<0.01$, ${ }^{\star \star \star} p$ $<0.001$ without adjustment for multiple comparisons, unpaired two-sided $t$-test. Source data are provided as a Source data file.

Likewise, model predictions of MNZ and MNZ metabolite concentrations were not significantly different from experimental results in cultures starting with a $0.001 \times \mathrm{Gv}: \mathrm{Li}$ ratio (extracellular MNZ: $p=0.255$, intracellular MNZ: $p=0.336$, acetamide: $p=$ 0.877 ), but predictions for extracellular MNZ, intracellular MNZ, and acetamide concentrations in cultures with a $1000 \times \mathrm{Gv}: \mathrm{Li}$ ratio did vary significantly from experimental data (Supplementary Fig. 4). The deviation of model predictions when $G v$ is initially dominant suggests that experimental investigation of detailed mechanisms of $G v$ interactions with MNZ is warranted (for example, the potential ability of $G v$ to externally degrade MNZ). Despite some deviation of peripheral model predictions from experimental measurements, the Gv:Li ratio-dependent trends were reproduced by the model. The dependency on initial culture ratios of $G v$ to $L i$ on growth suggests that non-target bacteria that sequester MNZ could significantly alter drug efficacy.

We observed some variation in the sensitivity (EC50) of $L i$ to MNZ. Variability in minimum inhibitory concentrations (MIC) estimations have been reported, as changes in culture conditions including incubation length and the inoculum effect can influence the apparent sensitivity of bacteria to antibiotic ${ }^{37,41}$. In addition, the sensitivity of Lactobacillus spp. and $G v$ to $\mathrm{MNZ}$ and their MICs are reported to range from 500 to $4000 \mu \mathrm{g} / \mathrm{ml}$ and 0.75 to $>256 \mu \mathrm{g} / \mathrm{ml}$, respectively ${ }^{42-44}$. To ascertain whether our results would be influenced by variation in $L i$ sensitivity to MNZ, we repeated the simulations over a range of EC50 values. To represent reported resistance of Lactobacillus spp. in vitro, we increased the EC50 value of $L i$ to be 10-fold higher than $G v$ $\left(\mathrm{EC} 50_{\mathrm{Li}}=4200 \mu \mathrm{g} / \mathrm{ml}\right)$. MNZ efficacy in inhibiting $G v$ growth was similarly decreased at low Gv:Li ratios (36.5\% max growth at $0.001 \times \mathrm{Gv}: \mathrm{Li}$ ) compared to high Gv:Li ratios (3.96\% max growth at $1000 \times$ Gv:Li, Supplementary Fig. 5a). Li had little to no susceptibility over the range of MNZ concentrations tested (Supplementary Fig. 5b). In addition, these EC50 values replicated trends in experimental data for growth kinetics (Supplementary Fig. 2d, h versus Supplementary Fig. 5c, d). These results support that the initial Gv:Li ratio-dependent trends in MNZ efficacy for inhibiting $G v$ growth are independent of $L i$ 's sensitivity to MNZ. 
a

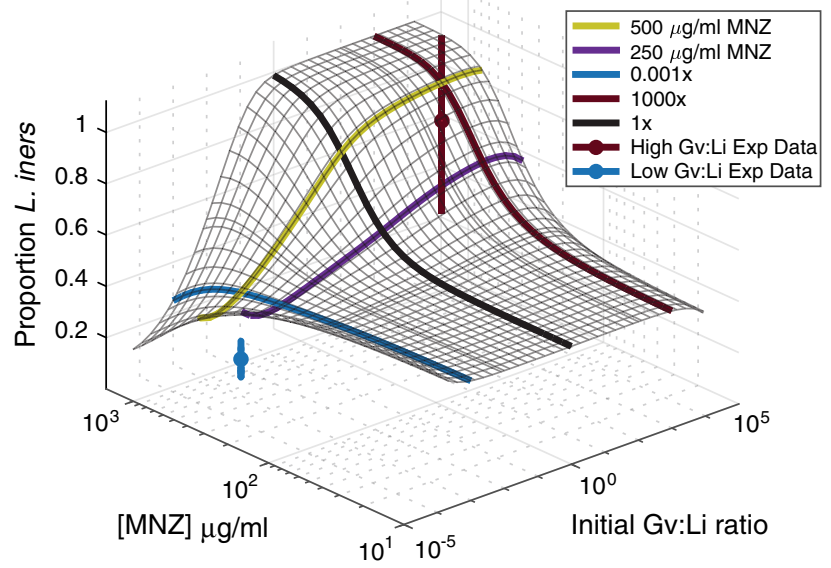

b

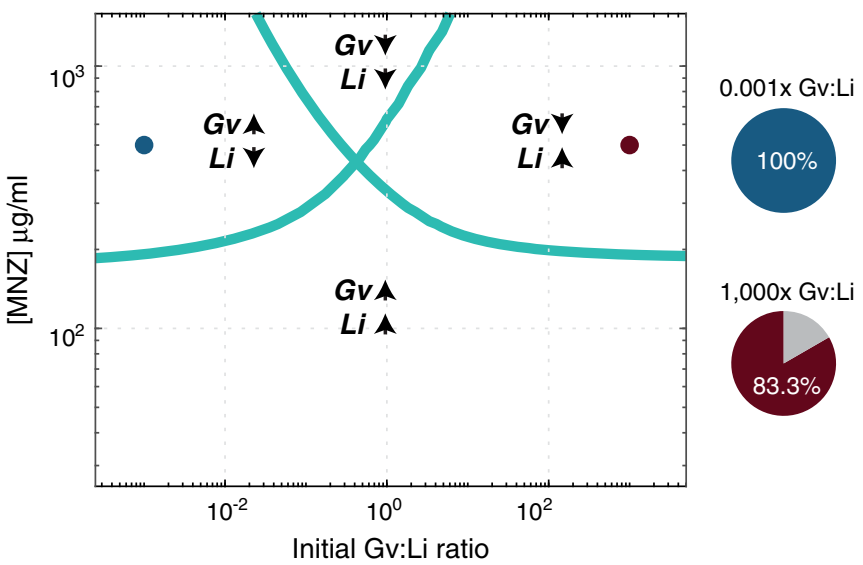

Fig. 3 Initial Gv:Li ratios dictate final microbial populations. a Surface plot illustrates model predictions for proportion of $L i$ relative to $G v 48 \mathrm{~h}$ at different starting Gv:Li ratios ( $x$-axis) and at different doses of MNZ ( $y$-axis). Experimental validation was performed in in vitro co-cultures of $L i$ and $G v$ ( $n=18$ independent, biological replicates for each ratio) and is plotted on the surface, with mean \pm SD represented by nodes and vertical lines. $\mathbf{b}$ Phase diagram of microbial growth dynamics $48 \mathrm{~h}$ after exposure to various MNZ doses, dots indicate experimental conditions evaluated. There are four possibilities: Both $\mathrm{Gv}$ and $\mathrm{Li}$ populations are increased after treatment, both Gv and Li populations are decreased, only the Gv population is increased and only the $\mathrm{Li}$ population is increased. Pie charts indicate the fraction of experimental samples that agree with the predicted trends (right).

Optimal MNZ doses are dependent on pre-treatment microbiome. We next used the model to determine specific combinations of MNZ concentrations and initial Gv:Li ratios that resulted in optimal final $L i$ proportion after $48 \mathrm{MNZ}$ exposure. The initial Gv:Li ratio was highly associated with the final Gv:Li ratio for doses of $\mathrm{MNZ}>250 \mu \mathrm{g} / \mathrm{ml}$ (Fig. 3a). Interestingly, cultures that were initially $L i$ dominant $(0.001 \times \mathrm{Gv}: \mathrm{Li})$, were nearly insensitive to any dose of $\mathrm{MNZ}$, resulting consistently with $>50 \% G v$ (Fig. 3a). This result carries the surprising implication that women with $L i$-dominant vaginal microbiomes at treatment initiation are likely to undergo recurrence, regardless of MNZ dose. Of note, cultures that were originally $G v$ dominant (Gv: $\mathrm{Li}$ $>1$ ) were the most likely to be $L i$ dominated after $48 \mathrm{~h}$ exposure to MNZ. Experimental data supported these trends, as the simulation predictions were not significantly different for the final proportion of $L i$ at $500 \mu \mathrm{g} / \mathrm{ml}$ for $1000 \times(p=0.680, t=0.420$, $\mathrm{df}=17)$. The model did overestimate the final proportion of $L i$ at the $0.001 \times \mathrm{Gv}: \mathrm{Li}$ ratio (predicting a $44.1 \%$ proportion of $\mathrm{Li}$ compared to $14.2 \% \pm 7.16 \%$ obtained experimentally); however, this result suggests an even more significant reduction in $\mathrm{Li}$ proportion when $G v$ is initially dominant $(p=0.008, t=4.06$, $\mathrm{df}=17)$.

A phase diagram of MNZ therapy outcomes at $48 \mathrm{~h}$ was created to characterize both $L i$ and $G v$ endpoint growth dynamics, which depict either an increase/expansion or decrease in population size relative to the initial population. The optimal growth dynamics would depict the expansion of only the $L i$ population and the least optimal growth dynamics would be the expansion of only $G v$. A decrease in both populations is additionally not optimal, as lower levels of beneficial microbiota are often associated with opportunistic infections or overgrowth of non-optimal species ${ }^{45,46}$. We observed that higher initial Gv:Li ratios in conjunction with MNZ concentrations over $250 \mu \mathrm{g} / \mathrm{mL}$ were more likely to result in optimal final growth dynamics where the $L i$ bacterial population was the only population expanding (Fig. 3b). Likewise, it was possible for only the $G v$ population to grow and the $L i$ population to decrease when the initial Gv:Li ratio was $<1 \times$. Interestingly, the diagram predicts that it is possible that both $G v$ and $L i$ populations would decrease for intermediate ratios of $\mathrm{Gv}: \mathrm{Li}$, which expand to include a wider range of ratios as the dose of $\mathrm{MNZ}$ is increased. Overall, in vitro co-culture experimental data supported the model predictions for endpoint growth dynamics, with 15 of 18 samples agreeing with the dynamics predicted by the phase diagram for the $1000 \times \mathrm{Gv}: \mathrm{Li}$, $500 \mu \mathrm{g} / \mathrm{ml}$ group and for all 18 samples agreeing with the predictions for the $0.001 \times \mathrm{Gv}: \mathrm{Li}$ ratio, $500 \mu \mathrm{g} / \mathrm{ml}$ group (Fig. $3 \mathrm{~b}$, right). This result reinforces the importance of pre-treatment Gv: $\mathrm{Li}$ ratio on post-treatment bacterial community composition.

Initial composition influences efficacy in more complex models. While our model results emphasize the importance of pretreatment Gv:Li ratios in MNZ efficacy in co-cultures, BV in women is more complex, and involves interspecies interactions and strain variability across many different bacterial species. To evaluate the above results in more complex settings that include multiple species, interspecies interactions, and strain variability, we created three additional model structures (Fig. 4a-d). In Model B and Model D, we account for potential interspecies interactions, such as amensalism between Lactobacillus spp. and $\mathrm{BV}$-associated bacteria and commensal or mutualistic behavior within BV-associated bacteria subpopulations and Lactobacillus spp. (Fig. 4a, d) ${ }^{47-51}$. In Models $C$ and $D$ we add additional representative species; a second $\mathrm{BV}$-associated species and second Lactobacillus spp. (Fig. 4c, d). To address potential variability in associated parameters, we randomly selected parameter values from physiologically relevant ranges determined from previously published studies (Supplementary Tables 2 and 3). Notably, across all four model structures we found that higher initial relative amounts of $\mathrm{BV}$-associated bacteria to Lactobacillus spp. had higher relative post-antibiotic levels of Lactobacillus spp. (BV: LB ratio, Fig. 4e, f, Supplementary Fig. 6, $p<1 \mathrm{E}-6, p<1 \mathrm{E}-6, p<$ $1 \mathrm{E}-6, p<1 \mathrm{E}-6)$. This result held for a range of ratios $(0.6 \times \mathrm{BV}: \mathrm{LB}$ and $100 \times \mathrm{BV}: \mathrm{LB})$ chosen to reflect the observed relative abundance of Lactobacillus spp. in BV positive women $(60-1.0 \%)^{52}$. Moreover, for each of these model structures, the global sensitivity analyses consistently selected the MNZ internalization/ sequestration parameter $\left(k_{\text {int }}\right)$ and the initial relative abundance of BV-associated bacteria to Lactobacillus spp. (BV:LB ratio) as significantly sensitive parameters in post-antibiotic treatment Lactobacillus spp. relative abundance. Variability in $G v$ sensitivity 
a

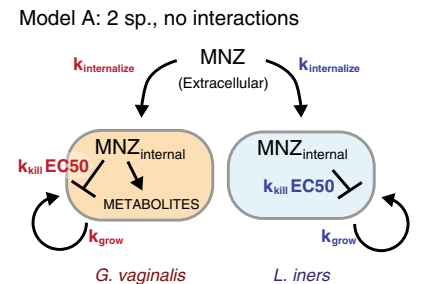

b

Model B: 2 sp., with interactions

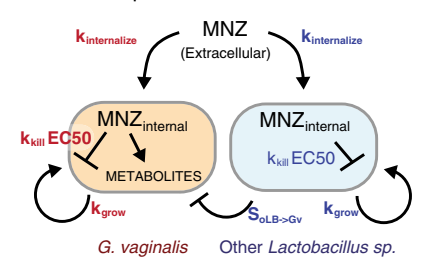

e

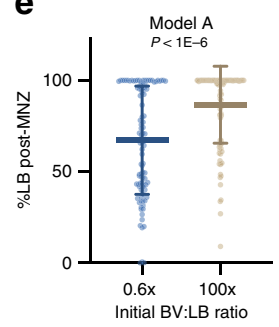

g

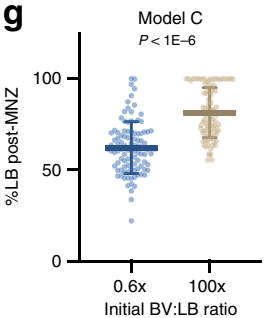

$\mathbf{f}$
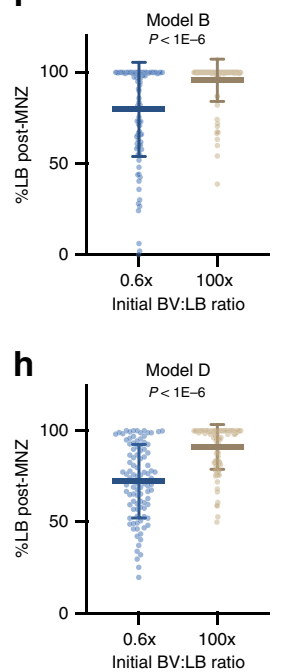

Model C: 4 sp., no interactions
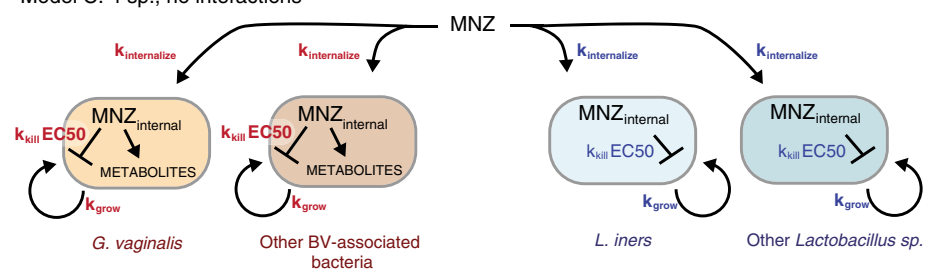

d

Model D: 4 sp., with interactions
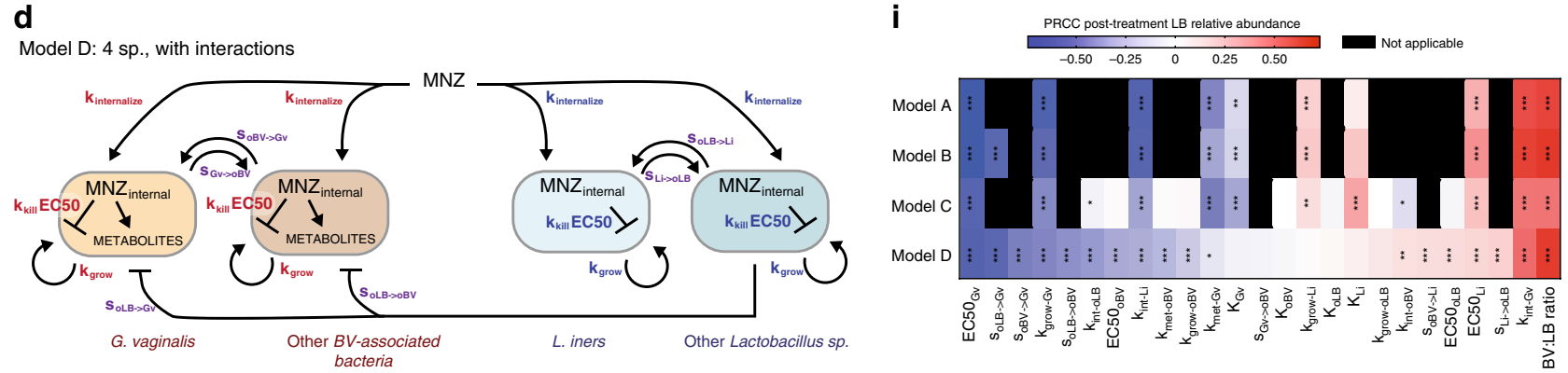

Fig. 4 High pre-treatment BV:LB ratio is predicted to reduce MNZ efficacy in more complex microbial environments regardless of strain variability. a Original model structure validated in Fig. 2. b Two-species model with negative interaction between other Lactobacillus spp. (oLB) and Gv. c Four species model of Gv and Li with additional representative bacteria for BV-associated bacteria and Lactobacillus spp. d Four species model with interspecies interactions. Within BV-associated bacteria and Lactobacillus spp. interactions were simulated from mutualistic (both benefit) to commensal (one benefits, the other is neutral). Inhibitory (amensal) interactions are included between D-lactic acid producing bacteria, other Lactobacillus spp., with both BVassociated bacteria. e-h Post-MNZ treatment $(48 \mathrm{~h}, 500 \mu \mathrm{g} / \mathrm{ml})$ Lactobacillus spp. relative abundances at $0.6 \times$ and $100 \times$ BV-associated bacteria to Lactobacillus spp (BV:LB) ratios for each model type $(n=100$ independent simulations for each ratio, data are presented as mean \pm SD). Each point represents a parameter set randomly sampled from physiological ranges in Supplementary Tables 2 and 3. Statistical analysis was completed using unpaired, two-sided $t$-tests: Model $A\left(p=7.20 \times 10^{-7}, t\right.$ ratio $\left.=5.32, \mathrm{df}=198\right)$, Model $\mathrm{B}\left(p=1.67 \times 10^{-7}, t\right.$ ratio $\left.=5.649, \mathrm{df}=198\right), \mathrm{Model} C(p=8.05 \times$ $10^{-18}, t$ ratio $\left.=9.725, \mathrm{df}=198\right)$, and Model $\mathrm{D}\left(p=1.70 \times 10^{-13}, t\right.$ ratio $\left.=7.954, \mathrm{df}=198\right)$, which were corrected for multiple comparisons using the Benjamini and Hochberg method. i Significantly sensitive parameters were assessed by partial rank correlation for each model structure (a-d) in a global sensitivity and uncertainty analysis, multiple comparisons where adjusted for using Bonferroni correction (asterisks indicate significance as ${ }^{\star} p<0.05,{ }^{\star *} p<$ $\left.0.01,{ }^{\star \star \star} p<0.001\right)$. Source data are provided as a Source data file.

to $\mathrm{MNZ}$ (EC50) and growth rate were also selected as critical parameters in dictating response to MNZ treatment, which are of interest as there is significant variability across $G v$ subclasses in terms of resistance to MNZ, and metabolism ${ }^{53}$. Furthermore, when models were modified such that Lactobacillus spp. could not internalize/sequester MNZ, the ratio-dependent effect was abrogated, and was independent of sensitivity of Lactobacillus spp. to MNZ (Supplementary Fig. 7a, b). Altogether, this provides additional quantitative evidence that Lactobacillus spp. sequestration of MNZ may contribute to BV recurrence in more complex microbial environments.

Pre-treatment composition is associated with clinical outcome. We next evaluated whether the influence of initial BV:LB ratio on MNZ efficacy is observed clinically. We compared the pretreatment ratio of $\mathrm{BV}$-associated bacteria to Lactobacillus spp.
(BV:LB ratio) in vaginal samples collected from women who underwent MNZ treatment for BV and were cured or experienced recurrence, in two clinical studies; the UMB-HMP study ${ }^{33}(n=$ 11) and CONRAD BV study ${ }^{34}(n=33)$. We chose to evaluate each study separately to minimize the effects of differences in sample collection and in methods of microbial species measurements. In the UMB-HMP cohort, 11 women were observed over the course of 10 weeks and provided cervicovaginal lavage (CVLs) samples each day for quantification of relative microbial abundances by sequencing of the V3 and V4 regions of 16S rRNA. Patients underwent treatment for BV that consisted of 1 week of 500-mg oral MNZ, taken twice daily. Of the 11 women, 8 met inclusion criteria and were classified as recurrent or cured dependent on Nugent scoring, where recurrent patients were described as women who responded to treatment but exhibited a second episode of BV during the 10-week period (Supplementary Table 4). Results resonated with model predictions where 

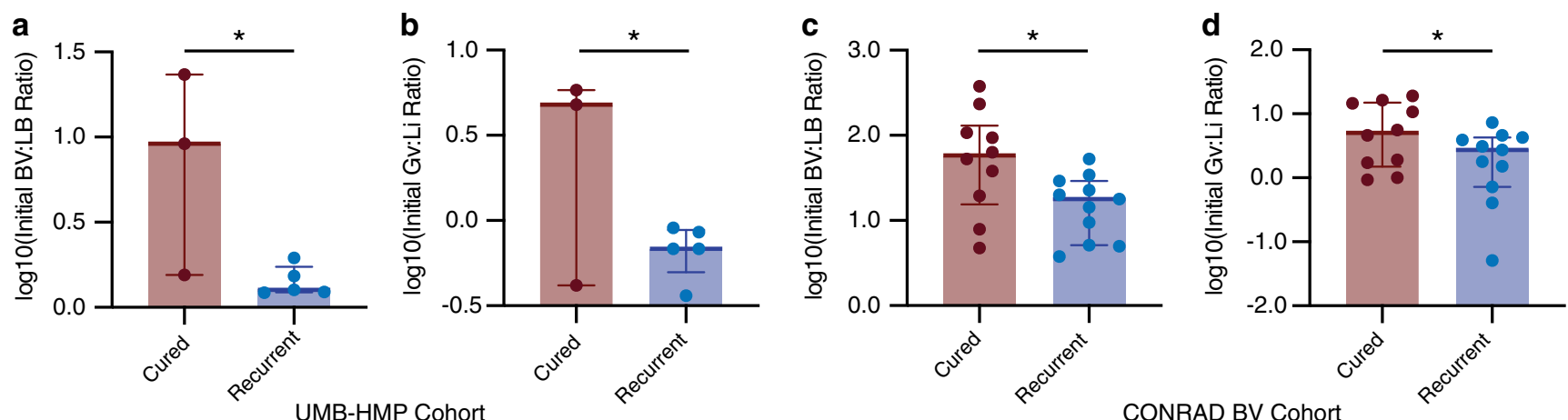

Fig. 5 Increased initial BV:LB ratios associated with successful treatment of BV. a, b Clinical results for the UMB-HMP cohort ( $n=3$ individuals for the cured group, $n=5$ individuals for the recurrent group) describing the a log base 10 transform of initial BV-associated bacteria relative abundance to Lactobacillus spp. relative abundance, $p=0.0366, t=2.678, \mathrm{df}=6$. b Initial Gv:Li ratio, $p=0.0497, t=2.451, \mathrm{df}=6$. c, $\mathbf{d}$ Clinical results for the CONRAD BV cohort ( $n=10$ individuals for the cured group, $n=11$ individuals for the recurrent group) describing $\mathbf{c}$ log base 10 transform of initial BV-associated bacteria relative abundance to Lactobacillus spp. relative abundance, $p=0.0242, t=2.449, \mathrm{df}=19 . \mathbf{d} \mathrm{Initial} \mathrm{Gv}: \mathrm{Li}$ ratio, $p=0.0338, t=2.287, \mathrm{df}=19$. Data are presented as median, 25th and 75th quartiles, statistical analysis was completed with unpaired, two-sided $t$-tests that were not adjusted for multiple comparisons. Source data are provided as a Source data file.

individuals who experienced recurrence had higher amounts of Lactobacillus spp. relative to BV-associated bacteria (lower BV:LB ratios, $p=0.0366$ ) at treatment initiation and tended to have higher abundances of Lactobacillus spp., particularly $L i$, but abundance of individual species were not statistically significant after adjustment for multiple comparisons $(p=0.201$, Fig. 5a, Supplementary Fig. 8a). In addition, $G v$ relative abundance was not significantly different between groups $(p=0.984$, Supplementary Fig. 8b). Furthermore, when we analyzed the specific species in the original two-species model, we also observed similar results where cured women had significantly higher ratios of $G v$ to $L i(p=0.0497$, Fig. $5 b)$. It is important to note that since the Gv:Li ratio comparison was a selective analysis, we did not correct for multiple comparisons based on individual species in the original data set (over 190 species measured). These results support both the in vitro experimental data and model results that predicted a lower efficacy of MNZ treatment when a lower ratio of $G v$ to $L i$ was present pre-treatment.

We also evaluated model findings in a second clinical cohort, the CONRAD BV study, which consisted of 33 women whose vaginal microbiome was sampled at enrollment in the study, 1 week after MNZ treatment and 1 month after MNZ treatment. Relative abundances were determined by sequencing of the V4 region of the $16 \mathrm{~S}$ rRNA. Women were excluded from this subset analysis if they failed to finish the antibiotic regimen, contracted a secondary vaginal infection, did not respond or had delayed response of treatment. Of the 33 women, 21 met inclusion criteria and were evaluated by molecular-BV criteria (dominance of Lactobacillus spp.) at 1 week and 1 month, with women exhibiting a vaginal microbiota composition of $<50 \%$ Lactobacillus spp. classified as BV positive. The group analyzed consisted of women who were cured $(n=10)$ and or were determined to have recurrent $\mathrm{BV}$ ( $n=11$; Lactobacillus was dominant at 1 week, but molecular-BV returned after 1 month, Supplementary Table 5). Like the previous study, we found that women who experienced recurrence had higher levels of Lactobacillus spp. relative to $\mathrm{BV}$-associated bacteria (lower $\mathrm{BV}: \mathrm{LB}$ ratio, $p=0.0242$, Fig. 5c). Comparison of CLR-transformed relative abundance did not result in statistically significant differences for $L i$ or $G v$, but tended to support the trend of recurrent women having higher $L i$ and lower $G v$ (Supplementary Fig. 8c, d, $p=0.521, p=0.694$ ). Similarly, analysis of the Gv:Li ratio supported higher pretreatment $G v$ relative to $L i$ was associated with better treatment outcomes (Fig. 5d; $p=0.0338$ ). Though preliminary and limited by low sample numbers, these results support the model predictions and suggest that successful BV treatment could be driven by competition for MNZ, where non-target bacterial populations, Lactobacillus spp., like $L i$ sequester MNZ away from target bacterial populations like $G v$, A. vaginae, Sneathia, etc., ultimately decreasing MNZ efficacy.

\section{Discussion}

Here we show a personalized tolerance mechanism that may contribute to $\mathrm{BV}$ recurrence and treatment failure. Our model illustrates how non-target bacteria, such as $L i$ or other Lactobacillus spp., may sequester antibiotic and lower the amount of MNZ available to target bacteria like $G v$. This model result implies that MNZ efficacy may be dependent on highly variable pre-treatment relative abundances of Lactobacillus spp. such as $L i$ to $\mathrm{BV}$-associated bacteria populations (BV:LB ratios) and raises the question of whether patients with higher levels of Lactobacillus spp. are more susceptible to recurrent BV than those with higher degrees of dysbiosis. Importantly, results from the model, in vitro experiments, and clinical data all point to a higher pretreatment $\mathrm{BV}$-associated bacteria population relative to Lactobacillus spp. as a driver of MNZ efficacy in inhibiting $G v$ growth and facilitating post-treatment Lactobacillus dominance. This study complements ongoing work in the search for drivers of BV treatment efficacy, in which experimental studies are often limited to delineating the role of individual bacteria, and it is challenging to assess the importance of numerous clinical and microbial variables that are associated with treatment outcome ${ }^{15}$.

The potential for non-antibiotic-target bacterial populations to act as a sink for MNZ and alter efficacy is similar to a concept that has been previously explored in bacterial ecology, termed the inoculum effect (IE), which describes an increase in antibiotic MICs due to increased bacterial load and decreased per cell antibiotic concentration ${ }^{54}$. While the IE and the ability of bacterial species to influence MNZ bioavailability has been previously reported, to our knowledge its role in BV recurrence has not yet been considered. Furthermore, the ODE model used here was essential for determining the critical importance of MNZ sequestration by Lactobacillus spp. across multiple interactions that have the potential to influence efficacy and recurrence, including metabolism, proliferation, and susceptibility to MNZ of both target and non-target species. The model was also necessary 
for translating the importance of this parameter to microbial communities with varying compositions and with different MNZ dosing regimens. Though the proposed MNZ sequestration mechanisms were not experimentally validated in this study, the model predictions for associated relationships between pretreatment microbial composition and BV recurrence were recapitulated in both co-cultures and in cervicovaginal samples, providing an additional mechanism for recurrence that has not previously been considered.

Recent studies evaluating pre-treatment vaginal microbiota composition on MNZ efficacy have reported inconsistent results, likely due to differences in patient exclusion criteria, time point of treatment outcome assessment, drug regimen, and methods to collect and quantify the vaginal microbiota. One study that employed a similar drug regimen (oral MNZ) and sample collection methods to the clinical cohorts evaluated here supported our results, finding higher pre-treatment loads of antibiotic-target species, $G v$ and A. vaginae, associated with BV treatment efficacy $^{19}$. Other studies that used different sample collection methods and antibiotic regimens did not explicitly evaluate the pre-treatment ratio of BV-associated bacteria to Lactobacillus spp.; generally suggested there was an association between total Lactobacillus relative abundance and successful treatment $\mathrm{t}^{15,55,56}$. Notably, some of these studies focused on analyzing treatment outcome immediately after antibiotic therapy was completed, and in some cases treatment failure was due to no response to therapy. We propose that recurrence and failure to respond to therapy likely arise from different factors, where recurrence is due to a collective bacterial population's resilience to antibiotic therapy and failure to respond is due to inherent resistance of BVassociated bacteria. Studies that have associated higher $G v$ loads with treatment failure correspond with the latter and could be due to the formation of biofilms or other resistance mechan$\mathrm{isms}^{56}$. As our model predicts immediate post-therapy Lactobacillus spp. relative abundance, no response to treatment would be equivalent to predicting no change or low Lactobacillus spp. relative abundance at $48 \mathrm{~h}$. An additional limitation of our model is that it does not appear to be applicable to cases of MNZ treatment failure in women who initially had very low levels of Lactobacillus spp. $(<1 \%)$, which our model would predict should promote MNZ efficacy ${ }^{34}$. However, we propose that treatment failure in this case may be a result of the Allee effect ${ }^{57,58}$, which can be caused by a variety of mechanisms that lead to decreased fitness at low population densities, suggesting these women have Lactobacillus abundances that are too low to recolonize the vagina and may be associated with more precisely modeling interspecies interactions. Moreover, since $L i$ is the only Lactobacillus spp. observed to date to significantly sequester MNZ, it will be important to characterize how other vaginal bacterial species interact with MNZ to further explore the role of non-target bacterial species on MNZ efficacy. Altogether, conflicting results in clinical studies of pre-treatment vaginal microbiota composition support the need for the development of quantitative platforms to evaluate the interplay between multiple microbial species, clinical variables, and dosing regimens that contribute to personalized differences in treatment failure.

Models presented here are only simple reconstructions of the minimal possible interactions between bacterial species and an antibiotic that have been established as key species by the existing literature $\mathrm{e}^{10,15,17}$, with a time-scale that was limited by in vitro coculture conditions. While the model provided useful insight into how non-target bacterial species may influence BV recurrence after MNZ treatment, predicting regrowth of Lactobacillus spp., and the full quantitative mechanisms underlying responses to treatment are likely more nuanced. More complex model frameworks did suggest key results would hold true in microbial communities with additional microbial species, interspecies interactions, and strain variability, though we were not able to validate this experimentally. Interspecies interactions in our models were incorporated with generalized Lotka-Volterra equations which simplifies relationships to a single term, but represent a good starting point for recapitulating ecosystem-level complexities ${ }^{59-63}$. Specific metabolic interactions that dictate survival and elimination of bacterial species in the vagina could be included with greater mechanistic detail in the future. In instances where parameters are unknown or difficult to measure experimentally, this work demonstrates the value of a global computational sensitivity analysis for understanding the relative importance of strain-level differences in antibiotic uptake, metabolism, or sensitivity. Predictive simulations can be run across multiple possible parameter ranges to determine the effects of variation prior to costly experimental measurements. This tool will be valuable in isolating the role of individual parameters in making a bacterial population or community more tolerant to antibiotic therapy.

In this study, we demonstrated that ODE models can provide insights into antibiotic-microbe interactions pertinent to understanding BV treatment efficacy. Our work highlights that it is possible for BV treatment to fail, even if target bacteria are not resistant to MNZ as vaginal bacterial populations as a whole can be resilient to antibiotic, resulting in recurrent BV. While our clinical analysis is limited in sample size and therefore should be considered preliminary, future extensions of this work could be used to inform clinical decision-making regarding personalized therapy options. More generally, we envision that the use of quantitative models such as this will provide a framework for integrating knowledge of interactions between multiple bacterial species and drug treatments in mucosal tissues to give insight into the diverse responses observed in infectious disease and other syndromes of the female reproductive tract.

\section{Methods}

Bacterial strains and culture conditions. Lactobacillus iners ATCC 55195 and Gardnerella vaginalis ATCC 14018 (group C) were obtained from the American Type Culture Collection (ATCC) and maintained on Human Bilayer Tween Agar (BD) plates and New York City III (NYCIII) medium according to the manufacturer's instructions. Agar plates and liquid cultures were incubated at $37^{\circ} \mathrm{C}$ with anaerobic gas mixture, $80 \% \mathrm{~N}_{2}, 10 \% \mathrm{CO}_{2}$, and $10 \% \mathrm{H}_{2}$. Frozen stocks of strains were stored at $-80{ }^{\circ} \mathrm{C}$ in $40 \%(v / v)$ glycerol.

\section{Metronidazole quantification by tandem mass spectrometry. MNZ con-} centrations were determined by validated LC-MS/MS assays. Sample aliquots were centrifuged at $3000 \times g$ and divided between supernatant and cell pellet. Extracellular MNZ was extracted from supernatant via protein precipitation using acetonitrile. For intracellular concentration measurements, cell pellets were lysed using sonication and re-suspended in $100 \mu \mathrm{L}$ of sterile water. Samples were subjected to positive electrospray ionization (ESI) and detected via multiple reaction monitoring (MRM) using a LC-MS/MS system (Agilent Technologies 6460 QQQ/ MassHunter). Calibration standards were prepared with an inter- and intra-day precision and accuracy of $\leq 5 \%$ with an $r^{2}$ value of $0.9988 \pm 0.0009$. Quantification was performed using MRM of the transitions of $\mathrm{m} / z \quad 172.2 \rightarrow 128.2$ and $176.2 \rightarrow$ 128.2 for MNZ and MNZ-d4 respectively. Each transition was monitored with a 100-ms dwell time. Stock solutions of MNZ and MNZ-d4 were prepared at $1 \mathrm{mg} /$ $\mathrm{mL}$ in acetonitrile-water and stored at $-20^{\circ} \mathrm{C}$. Mobile phase $\mathrm{A}$ is $0.1 \%$ acetic acid in $\mathrm{H}_{2} \mathrm{O}$ and mobile phase $\mathrm{B}$ is $0.1 \%$ acetic acid in $\mathrm{ACN}$, and chromatographic separation was achieved using a gradient elution with a Chromolith Performance $\mathrm{RP}-\mathrm{C} 18$ column maintained at $25^{\circ} \mathrm{C}$ from 0 to $4.6 \mathrm{~min}, \mathrm{~B} \% 0-100$, with $0.5 \mu \mathrm{L} / \mathrm{min}$ flow. During pre-study validation, calibration curves were defined in multiple runs on the basis of triplicate assays of spiked media samples as well as QC samples. This method was validated for its sensitivity, selectivity, accuracy, precision, matrix effects, recovery, and stability. Replicates of reference samples were included every six samples and evenly distributed throughout the MS analysis to monitor consistency and performance and to utilize for downstream normalization.

Bacterial quantification. Bacterial quantification determined via turbidimetry was completed by measuring the optical density at each time point, $100 \mu \mathrm{L}$ of sample inoculum was read at O.D. $600 \mathrm{~nm}$ using a SpectraMax Plus 384 UV 
spectrophotometer. Time points were recorded within 5 min of sampling and stored at $4^{\circ} \mathrm{C}$

Bacterial quantification using plate counting was done by doing a 10 -fold dilution using sterile water and aliquoting $100 \mu \mathrm{L}$ spread evenly onto BD agar plates. Cultures were incubated at $37^{\circ} \mathrm{C}$. Plating was done in triplicates and was counted manually. Prior optimization ensured the dilution would result in no more than 300 colonies making quantification as accurate as possible.

For co-culture validation experiments, $100 \mu \mathrm{L}$ of sample was aliquoted on Rogosa agar and Gardnerella selective agar. Experiments were conducted to verify the absence of Lactobacillus growth on Gardnerella selective media and absence of $G$. vaginalis growth on Rogosar agar, to confirm that colony formation specific to respective taxa. Cultures were incubated at $37^{\circ} \mathrm{C}$, with a total of 36 biological replicates for the $1000 \times$ and $0.001 \mathrm{Gv}$ :Li ratio cultures $(n=18$ cultures for each ratio). Plating was done in triplicates and was counted manually. Prior optimization ensured the dilution would result in no more than 300 colonies making quantification as accurate as possible.

Bacteria-MNZ experiments. For the MNZ experiments, $50 \mu \mathrm{L}$ MNZ was added at appropriate concentrations to $5 \mathrm{~mL}$ of NYCII media. Samples equilibrated at $37^{\circ} \mathrm{C}$ for $1 \mathrm{~h}$ prior to the addition of $50 \mu \mathrm{L}$ of bacterial inoculum $\left(2 \times 10^{6}\right)$. One hundred and fifty microliters of aliquot was taken for time-point readings for MNZ and bacterial quantification (as described above). Samples were incubated at $37^{\circ} \mathrm{C}$ under constant mixing and only removed for time-point measurements.

For the co-culture experiments, Gv:Li ratios were added at appropriate experimental conditions in a likewise manner. For each varying ratio sample within each experiment, a side-by-side duplicate was performed without MNZ as a negative control. The negative control was assessed only for bacterial quantification to ensure that no growth condition or external stimuli promoted the growth of one over another. Negative control experiments demonstrated bacterial proliferation that modeled growth curves of each individual bacterium cultured alone thus confirming any changes in growth seen in our bacteria-MNZ experiments were the result of the addition of MNZ.

ODE models. The model equations were constructed assuming both $L i$ and $G v$ internalize MNZ at rates $k_{\text {int-Li }}$ and $k_{\text {int-Gv }}$, MNZ toxicity to $L i$ and $G v$ occurred at rates dependent on the maximum rates $k_{\text {kill-Li }}$ and $k_{\text {kill-Gv }}$ and the concentration of internalized MNZ where growth inhibition increased as internalized MNZ exceeded a threshold as described by $50 \%$ effective concentrations, EC $50_{\mathrm{Li}}$ and $\mathrm{EC} 50_{\mathrm{Gv}}$. The growth of $L i$ and $G v$ was assumed to be logistic in behavior at rates $k_{\text {grow-Li }}$ and $k_{\text {grow-Gv }}$ with distinct carrying capacities for each bacterium, $K_{\mathrm{Li}}$ and $K_{\mathrm{Gv}}$. The parameters for $k_{\text {grow-Li, }} k_{\text {grow-Gv }}, K_{\mathrm{Li}}$, and $K_{\mathrm{Gv}}$ were determined by nonlinear leastsquares fitting of the logistic function to growth curves for $L i$ and $G v$ grown in separate cultures (Supplementary Fig. 2a, b) ${ }^{64}$. The $k_{\text {kill-Li, }} k_{\text {kill-Gv }}, \mathrm{EC} 50_{\mathrm{Li}}$, and EC50 $0_{\mathrm{Gv}}$ were determined by fitting the Hill equation to kill curves for $L i$ and $G v$ cultured in isolation (Supplementary Fig. $2 c$, d). Internalization rates, $k_{\text {int-Li }}$ and $k_{\text {int-Gv }}$ and metabolism rates, $k_{\text {acet }}$ and $k_{\text {met }}$ were determined from fitting the ODE model to time course mass spectrometry data for external MNZ, internal MNZ and acetamide and cell densities (optical density) using a multistart local optimization strategy (Multistart) with the local solver lsqcurvefit.

Model simulations and validation. Unless otherwise noted, all simulations were completed at MNZ concentration of $500 \mu \mathrm{g} / \mathrm{ml}$ over the course of $48 \mathrm{~h}$. Growth outputs were normalized to the maximal growth density $\left(K_{\mathrm{Li}}\right.$ and $\left.K_{\mathrm{GV}}\right)$ for comparison across simulations and to experimental data. External MNZ, internal MNZ, and acetamide concentrations were relative to the total volume of cellular pellets. Sensitivity analyses were completed by perturbing a single model parameter while keeping the rest of the parameters constant over $1000 \times-0.001 \times$ the original value. Surfaces were generated over three orders of magnitude for MNZ concentration $(10-1500 \mu \mathrm{g} / \mathrm{ml})$ and eight orders of magnitude for ratio of Gv:Li $\left(1.6 \times 10^{-4}-1.6 \times\right.$ $\left.10^{4}\right)$ at 1225 combinations of MNZ concentration and Gv:Li ratio. Model validation was completed by comparing the experimental co-culture data to model predictions using unpaired $t$-tests.

Generalized models and global sensitivity analysis. To incorporate intraspecies and interspecies variation we developed three additional model structures and ran simulations with randomized parameter sets to determine if the influence of initial Gv:Li ratio, or the more generalized BV:LB ratio, on endpoint Lactobacillus spp. composition is consistently observed across these model structures.

For capturing intraspecies variation, we used Latin Hypercube Sampling of parameter ranges for each parameter to create 100 parameter sets. We derived these parameter ranges from the literature and a summary of these ranges can be found in Supplementary Tables 2 and 3. These same parameter ranges and sampling methods were used for the global sensitivity and uncertainty analysis, which analyzed the partial rank correlation coefficient with 2000 randomly generated parameter sets with endpoint ( $48 \mathrm{~h}, 500 \mu \mathrm{g} / \mathrm{ml} \mathrm{MNZ)} \mathrm{Lactobacillus} \mathrm{spp.}$ relative abundance ${ }^{65}$. For capturing interspecies variation, and microbe-microbe interactions like cross-feeding, we developed a four species model that includes two representative BV-associated bacteria, and two Lactobacillus species, L. iners and a second species representing $L$. crispatus, L. jensenii, or L. gasseri.
Internalization/uptake rates $\left(\mathrm{k}_{\text {int }}\right)$. To our knowledge, this is the first publication that demonstrates that G. vaginalis and L. iners uptake or sequester MNZ. Previous literature describing uptake of MNZ in other bacterial species, including both obligate and facultative anaerobes has been published by Ralph and Clarke ${ }^{31}$, Tally et al. ${ }^{27}$, and Narikawa ${ }^{66}$. These publications demonstrate that even bacteria that are resistant to MNZ can still uptake MNZ, and at similar rates. Despite the fact that facultative anaerobes are believed to be largely insensitive to MNZ, Narikawa specifically demonstrates that nitroreductase activity is associated with the ability to uptake MNZ, and that pyruvate:ferrodoxin activity is associated with sensitivity to $\mathrm{MNZ}$ as an explanation for why the facultative anaerobes Escherichia coli, $K$. pneumoniae, M. morganii, and S. faecalis exhibited high MICs, but reduced supernatant MNZ. We calculated the rates of MNZ uptake for five species, one obligate anaerobe, B. fragilis, and four facultative anaerobes (E. coli, S. aureus, P. morganii, and S. faecalis) by digitizing the kinetic data for cell counts and extracellular MNZ concentrations in Ralph and Clarke ${ }^{31}$ and fitting second-order reaction kinetics by ordinary least-squares regression. The rates ranged from $2 \times 10^{-17}$ to 0.15 cell density ${ }^{-1} \mathrm{~h}^{-1}$. To determine the likelihood that these parameters could be a basis for Lactobacillus spp. uptake of MNZ, we assessed the similarity between $E$. coli's oxygen-independent NADPH-nitroreductase, nsfA, with nitroreductase protein sequences of $G$. vaginalis (34.7\%), L. crispatus (31.0\%), L. iners (29.4\%), L. jensenii (19.4\%), and L. gasseri (18.52\%). In addition, Guillen et al. ${ }^{67}$ reported that $L$. plantarum had selective nitroreductase activity, that shared $32-43 \%$ sequence similarity with several Lactobacillus species, and in comparison had similarity with $G$. vaginalis (24.0\%), L. crispatus (38.5\%), L. iners (25.5\%), L. jensenii (52.8\%), and L. gasseri (30.0\%). Sequence similarity was assessed by NCBI's protein BLAST ${ }^{68}$. As obligate anaerobes were observed to uptake MNZ at higher rates, we assumed that the other $\mathrm{BV}$-associated bacteria, which could be an obligate anaerobe could potentially have higher capacity to internalize MNZ.

Growth rates $\left(\mathrm{k}_{\text {grow }}\right)$ and carrying capacities $(\mathrm{K})$. To account for potential variability in growth rates, we surveyed previously published to determine ranges in growth For Lactobacillus species, we calculated growth rates by digitizing growth curves from Chetwin et al. ${ }^{69}$ and analyzed growth rates reported in Juárez-Tomás ${ }^{70}$, Anukam and Reid ${ }^{45}$. G. vaginalis and other bacterial strains growth curves were less abundant in the literature, but we did calculate growth rates from Atassi et al. ${ }^{49}$ and Anukam and Ried ${ }^{45}$. Generally, G. vaginalis and other BV-associated bacteria seemed to have slower growth rates than Lactobacillus species, and in the same culture conditions this was observed in Anukam and Ried ${ }^{45}$. For carrying capacity we assumed that there were similar carrying capacities for all species, except the $\mathrm{BV}$-associated bacteria based on data from Castro et al..$^{71}$, that reported $A$. vaginae at lower levels that $G$. vaginalis at steady state ${ }^{71}$.

Sensitivity to MNZ (EC50 and $\mathrm{k}_{\text {kill }}$ ). MNZ is highly variable, and typically obligate anaerobes are considered the most sensitive to MNZ. The strain of G. vaginalis used in the basis of this model is relatively resistant to MNZ, with growth barely inhibited at $256 \mu \mathrm{g} / \mathrm{ml}$ (9\% inhibition compared to $0 \mu \mathrm{g} / \mathrm{ml}$ control, Supplementary Fig. 2d). For A. vaginae, the MIC can range $2-256 \mu \mathrm{g} / \mathrm{ml}$ and $\mathrm{G}$. vaginalis can range from 0.75 to $>500 \mu \mathrm{g} / \mathrm{ml}^{43,44}$. Generally, it is assumed that Lactobacillus spp. are insensitive to MNZ; however, this also appears to be highly strain and speciesdependent with some Lactobacillus isolates in similar ranges of sensitivity as $G$. vaginalis $^{42,45}$. The rate at which MNZ inhibits growth is more difficult to find, as the experiments to determine this rate are more laborious than the standard kill curve to calculate EC50 so we assumed all kill rates to be equal across all species.

Metabolism of MNZ. To our knowledge, this is the first manuscript to describe the metabolism of MNZ by vaginal microbiota. We solely based the parameter value on the rate observed for the G. vaginalis strain from the model. In addition, we assumed that only BV-associated bacteria metabolism MNZ based on the observation that only $\mathrm{BV}$-associated bacteria metabolize HIV microbicide drugs ${ }^{29}$.

Interspecies interaction terms. Gause ${ }^{72}$ noted the calculation for interaction terms for a generalized Lotka-Volterra model describing competitive exclusion (Eqs. (1) and (2)). In our model, we generalized the interaction terms further to be able to capture many different interactions, specifically amensal behavior where Lactobacillus spp. can inhibit BV-associated bacterial growth with no effect of BVassociated bacteria on Lactobacillus species growth $(-/ 0)$ as well as mutualistic (both species benefit from the other $+/+$ ) and commensal behaviors (one species benefits $0 /+$ ) between $\mathrm{BV}$-associated bacteria or within the Lactobacillus population. The amensal behavior between Lactobacillus species has been documented experimentally in co-culture ${ }^{73}$ and we calculated the interaction term for many different species and strains of Lactobacillus on G. vaginalis and Prevotella bivia from Atassi et al. ${ }^{50}$. It is largely believed that $\mathrm{D}$-lactic acid produced by many Lactobacillus species inhibits the growth of BV-associated bacteria; however, $L$. iners does not produce this isomer of lactic acid and is the reasoning behind not including an interaction term between $L$. iners and the BV-associated bacteria ${ }^{48,74}$. It is believed that commensal behavior exists between $G$. vaginalis and P. bivia in the form of cross-feeding, so we allowed the model to simulate this behavior ${ }^{51}$. In addition, G. vaginalis is associated with promoting the growth of other BVassociated bacteria like A. vaginae ${ }^{71}$. Calculations were completed assuming the reported mono and co-cultures were at steady state to derive Eqs. (3) and (4). 
Equations (3) and (4) relate to the parameters in Supplementary Table 3 by Eqs. (5) and (6), which generalizes the reported interaction strength from the literature to be able to be adjusted for varying carrying capacities simulated in the model that do not equal the carrying capacities from the literature.

$$
\begin{gathered}
\frac{d N}{d t}=r_{N} N\left[1-\frac{N+s_{\mathrm{P} \rightarrow \mathrm{N}} P}{K_{N}}\right] \\
\frac{d P}{d t}=r_{P} P\left[1-\frac{P+\mathrm{s}_{\mathrm{N} \rightarrow \mathrm{P}} N}{K_{P}}\right] \\
\mathrm{s}_{\mathrm{P} \rightarrow \mathrm{N}}=\left[\frac{K_{N}-N}{P}\right] \\
\mathrm{s}_{\mathrm{N} \rightarrow \mathrm{P}}=\left[\frac{K_{P}-P}{N}\right] \\
s_{\mathrm{P} \rightarrow \mathrm{N}}=\left[\frac{K_{N}-\mathrm{f}_{\mathrm{P} \rightarrow \mathrm{N}} K_{N}}{f_{N \rightarrow P} K_{P}}\right] \\
\mathrm{s}_{\mathrm{N} \rightarrow \mathrm{P}}=\left[\frac{K_{P}-f_{N \rightarrow P} K_{P}}{f_{P \rightarrow N} K_{N}}\right]
\end{gathered}
$$

Software. Parameterization, ODE modeling, and sensitivity analyses were completed using Matlab ${ }^{\circledR}$ 2018b (Matlab, Natick, MA). Statistical analyses were performed using PRISM 8, exact $p$-values $<1 \mathrm{E}-6$ were calculated in Matlab.

\section{Clinical data and study population}

The UMB-HMP cohort. The study results and associated clinical data were previously published ${ }^{33}$ and all data provided were de-identified to this study. The UMB-HMP study was not an interventional study, but an observational prospective study, where treatment information was recorded during a clinical exam at week 5 and week 10 for 135 nonpregnant women of reproductive age. Within this study, MNZ treatment was provided as standard of care, as recommended by the CDC (metronidazole $500 \mathrm{mg}$ orally twice a day for 7 days) ${ }^{75}$. The original study protocol was approved by the Institutional Review Board of the University of Alabama at Birmingham and the University of Maryland School of Medicine. Written informed consent was appropriately obtained from all participants, who also provided consent for storage and use in future research studies related to women's health.

Women self-collected cervicovaginal swabs for 10 weeks. Vaginal microbiota data were generated by sequencing the V3-V4 regions of the $16 \mathrm{~S}$ rRNA gene and is available in dbGAP BioProject PRJNA208535. In this study, the vaginal microbiota composition data from 11 women who experienced $\mathrm{BV}$ and were treated with MNZ during the UMB-HMP study were analyzed. Any participants who failed to complete the MNZ regimen, who did not have BV according to Nugent scoring at the time of MNZ treatment, or who did not have follow-up data available were excluded from the analysis. The initial relative abundances were averaged across the week before starting MNZ treatment. Patients were classified to have recurrent BV if they exhibited a second episode of BV based on Nugent scoring (7-10) during remaining of the 10 -week observation period.

The CONRAD BV cohort. The study results and associated clinical data were previously published ${ }^{34}$ and all data provided were de-identified to this study. The original clinical study protocol was approved by the Chesapeake Institutional Review Board (IRB) (Pro \#00006122) with a waiver of oversight from the Eastern Virginia Medical School (EVMS) and registered in ClinicalTrials.gov (\#NCT01347632). A total of 69 women were screened from symptomatic discharge and 35 women were enrolled in the study. Vaginal microbiota data were generated by sequencing the V4 region of the $16 \mathrm{~S}$ rRNA gene, providing taxonomic resolution at the general level.

Thirty-three women completed all three visits. BV was evaluated by vaginal microbiota compositional data (molecular-BV) ${ }^{76}$. After biological samples were obtained at visit 1 (V1), women with BV were prescribed twice daily, 500-mg MNZ for 7 days. Participants returned for visit 2 (V2) 7-10 days after completing the course of MNZ therapy and visit 3 (V3) 28-32 days after completing treatment. At all three visits, samples were obtained to evaluate vaginal semen (ABAcard, West Hills, CA), vaginal $\mathrm{pH}$, gram stain for Nugent score and semiquantitative vaginal flora culture. CVLs were collected, followed by vaginal swabs and three fullthickness biopsies.

Analysis of clinical outcomes. In the Human Microbiome Project cohort, patients were defined as cured or recurrent based on whether after initial MNZ treatment the patient suffered an additional episode of BV (Nugent 7-10) during the 10-week course of data collection. For analysis, initial flora relative abundances were averaged across the 7 days prior to reported treatment start date. To analyze the relative ratio between $\mathrm{BV}$-associated bacteria and Lactobacillus spp., we combined the relative abundances for the top $20 \mathrm{BV}$-associated bacteria and all Lactobacillus spp. The genera BV-associated bacteria included were Gardnerella, Atopobium, Megasphaera, BVAB1-3, Streptococcus, Prevotella, Leptotrichia, Anaerococcus,
Peptoniphilus, Eggerthella, Veillonella, Sneathia, Mobiluncus, Corynebacterium, Ureaplasma, Eubacterium, Porphyromonas, Dialister, Peptostreptococcus, Bacteroides, Fusobacterium, Actinomyces, and Bifidobacterium. Before statistical analysis, the BV:LB ratio was log-transformed, and the relative abundances of $L$. iners, $G$. vaginalis were center-log ratio (CLR) transformed, with pseudocounts added to taxonomic units with relative abundances equal to zero. Statistical analysis of the BV:LB ratio and Gv:Li ratio was completed using two-sided unpaired Student's $t$ tests and analysis of the CLR-transformed single species abundances was completed using two-sided unpaired Student's $t$-tests and were corrected using the FDR method of Benjamini and Hochberg (PRISM 8).

For the CONRAD BV cohort, treatment outcome was defined based on Lactobacillus dominance evaluated at enrollment, 7 days after treatment and 28-32 days after treatment. Patients that exhibited Lactobacillus dominance at both 1 week and 1 month after treatment were considered cured, and patients that exhibited Lactobacillus dominance only at week 1 and not at 1 month were considered recurrent. The statistical analysis followed the same methodology as the HMP cohort.

Reporting summary. Further information on research design is available in the Nature Research Reporting Summary linked to this article.

\section{Data availability}

The source data are provided with this paper for Figs. 2-5, Supplementary Figs. 1, 2, 4 and 8 , which includes the in vitro validation, model parameterization, and clinical validation. For the clinical studies, the UMB-HMP cohort study sequence data and metadata were deposited in the Sequence Read Archive (SRA; http://www.ncbi.nlm.nih. gov/Traces/sra/) under BioProject PRJNA208535 (The daily dynamics of the vaginal microbiota before and after bacterial vaginosis) ([SRP026107] and [SRA091234] $)^{33}$. An abbreviated data set necessary for the reproduction of Fig. 5a, b and Supplementary Fig. $8 \mathrm{a}, \mathrm{b}$ are in Supplementary Table 4 and the Source data file. The sequence data and metadata for the CONRAD BV study are not in a formal repository, but are fully available upon request; however, we have included an abbreviated version of this data set that includes all the data necessary for reproduction of Fig. $5 c, d$ and Supplementary Fig. $8 c, d$ are in Supplementary Table 5 and can be found in the Source data file. Source data are provided with this paper.

\section{Code availability}

The code used to generate the model simulations is published (https://doi.org/10.5281/ zenodo.4121904) ${ }^{77}$.

Received: 26 August 2020; Accepted: 28 October 2020; Published online: 01 December 2020

\section{References}

1. Koumans, E. et al. The prevalence of bacterial vaginosis in the United States, 2001-2004; associations with symptoms, sexual behaviors, and reproductive health. Sex. Transm. Dis. 34, 864-869 (2007).

2. Peebles, K., Velloza, J., Balkus, J. E., McClelland, R. S. \& Barnabas, R. V. High global burden and costs of bacterial vaginosis: a systematic review and metaanalysis. Sex. Transm. Dis. 46, 304-311 (2019).

3. Taha, T. et al. Bacterial vaginosis and disturbances of vaginal flora: association with increased acquisition of HIV. Aids 12, 1699-1706 (1998).

4. Haggerty, C. L., Hillier, S. L., Bass, D. C. \& Ness, R. B. Bacterial vaginosis and anaerobic bacteria are associated with endometritis. Clin. Infect. Dis. 39, 990-995 (2004).

5. Hillier, S. L. et al. Association between bacterial vaginosis and preterm delivery of a low-birth-weight infant. The Vaginal Infections and Prematurity Study Group. N. Engl. J. Med. 333, 1737-1742 (1995).

6. Bradshaw, C. S. \& Brotman, R. M. Making inroads into improving treatment of bacterial vaginosis-striving for long-term cure. BMC Infect. Dis. 15, 292 (2015).

7. Ness, R. B. et al. A cluster analysis of bacterial vaginosis-associated microflora and pelvic inflammatory disease. Am. J. Epidemiol. 162, 585-590 (2005).

8. Nunn, K. L. \& Forney, L. J. Unraveling the dynamics of the human vaginal microbiome. Yale J. Biol. Med. 89, 331-337 (2016).

9. Fredricks, D. N., Fiedler, T. L., Thomas, K. K., Mitchell, C. M. \& Marrazzo, J. $M$. Changes in vaginal bacterial concentrations with intravaginal metronidazole therapy for bacterial vaginosis as assessed by quantitative PCR. J. Clin. Microbiol. 47, 721-726 (2009).

10. Srinivasan, S. et al. Temporal variability of human vaginal bacteria and relationship with bacterial vaginosis. PLoS ONE 5, e10197 (2010).

11. Bradshaw, C. S. et al. High recurrence rates of bacterial vaginosis over the course of 12 months after oral metronidazole therapy and factors associated with recurrence. J. Infect. Dis. 193, 1478-1486 (2006). 
12. Bradshaw, C. S. \& Sobel, J. D. Current treatment of bacterial vaginosislimitations and need for innovation. J. Infect. Dis. 214, S14-S20 (2016).

13. Ma, L., Su, J., Su, Y., Sun, W. \& Zeng, Z. Probiotics administered intravaginally as a complementary therapy combined with antibiotics for the treatment of bacterial vaginosis: a systematic review protocol. BMJ Open 7, e019301 (2017).

14. Ferris, D. G., Litaker, M. S., Woodward, L., Mathis, D. \& Hendrich, J. Treatment of bacterial vaginosis: a comparison of oral metronidazole, metronidazole vaginal gel, and clindamycin vaginal cream. J. Fam. Pract. 41, 443-449 (1995).

15. Xiao, B. et al. Association analysis on recurrence of bacterial vaginosis revealed microbes and clinical variables important for treatment outcome. Front. Cell. Infect. Microbiol. 9, 189 (2019).

16. Brotman, R. M. et al. A longitudinal study of vaginal douching and bacterial vaginosis - a marginal structural modeling analysis. Am. J. Epidemiol. 168, 188-196 (2008).

17. Wang, B. et al. Molecular analysis of the relationship between specific vaginal bacteria and bacterial vaginosis metronidazole therapy failure. Eur. J. Clin. Microbiol. Infect. Dis. 33, 1749-1756 (2014).

18. Zhou, X. et al. Recent advances in understanding the microbiology of the female reproductive tract and the causes of premature birth. Infect. Dis. Obstet. Gynecol. https://www.hindawi.com/journals/idog/2010/737425/abs/ (2010).

19. Ferreira, C. S. T. et al. Treatment failure of bacterial vaginosis is not associated with higher loads of Atopobium vaginae and Gardnerella vaginalis. J. Med. Microbiol. 66, 1217-1224 (2017).

20. Coleman, J. S. \& Gaydos, C. A. Molecular diagnosis of bacterial vaginosis: an update. J. Clin. Microbiol. 56, e00342-18 (2018).

21. Gajer, P. et al. Temporal dynamics of the human vaginal microbiota. Sci. Transl. Med. 4, 132ra52 (2012).

22. Tamarelle, J. et al. Non-optimal vaginal microbiota after azithromycin treatment for Chlamydia trachomatis infection. J. Infect. Dis. https://doi.org/ 10.1093/infdis/jiz499 (2019).

23. Vaneechoutte, M. Lactobacillus iners, the unusual suspect. Res. Microbiol. 168, 826-836 (2017).

24. Faust, K., Lahti, L., Gonze, D., de Vos, W. M. \& Raes, J. Metagenomics meets time series analysis: unraveling microbial community dynamics. Curr. Opin. Microbiol. 25, 56-66 (2015).

25. Lugo-Martinez, J., Ruiz-Perez, D., Narasimhan, G. \& Bar-Joseph, Z. Dynamic interaction network inference from longitudinal microbiome data. Microbiome 7, 54 (2019).

26. Brotman, R. M., Ravel, J., Cone, R. A. \& Zenilman, J. M. Rapid fluctuation of the vaginal microbiota measured by Gram stain analysis. Sex. Transm. Infect. 86, 297-302 (2010)

27. Tally, F. P., Goldin, B. R., Sullivan, N., Johnston, J. \& Gorbach, S. L. Antimicrobial activity of metronidazole in anaerobic bacteria. Antimicrob. Agents Chemother. 13, 460-465 (1978).

28. Church, D. L., Rabin, H. R. \& Lalshley, E. J. Role of hydrogenase 1 of clostridium pasteurianum in the reduction of metronidazole. Biochem. Pharmacol. 37, 1525-1534 (1988).

29. Klatt, N. R. et al. Vaginal bacteria modify HIV tenofovir microbicide efficacy in African women. Science 356, 938-945 (2017).

30. Edwards, D. I. Mechanisms of selective toxicity of metronidazole and other nitroimidazole drugs. Br. J. Vener. Dis. 56, 285-290 (1980).

31. Ralph, E. D. \& Clarke, D. A. Inactivation of metronidazole by anaerobic and aerobic bacteria. Antimicrob. Agents Chemother. 14, 377-383 (1978).

32. Edwards, D. I., Thompson, E. J., Tomusange, J. \& Shanson, D. Inactivation of metronidazole by aerobic organisms. J. Antimicrob. Chemother. 5, 315-316 (1979).

33. Ravel, J. et al. Daily temporal dynamics of vaginal microbiota before, during and after episodes of bacterial vaginosis. Microbiome 1, 29 (2013).

34. Thurman, A. R. et al. Bacterial vaginosis and subclinical markers of genital tract inflammation and mucosal immunity. AIDS Res. Hum. Retroviruses 31, 1139-1152 (2015).

35. Koch, R. L., Chrystal, E. J., Beaulieu, B. B. \& Goldman, P. Acetamide-a metabolite of metronidazole formed by the intestinal flora. Biochem. Pharmacol. 28, 3611-3615 (1979).

36. Regoes, R. R. et al. Pharmacodynamic functions: a multiparameter approach to the design of antibiotic treatment regimens. Antimicrob. Agents Chemother. 48, 3670-3676 (2004).

37. Meredith, H. R. et al. Applying ecological resistance and resilience to dissect bacterial antibiotic responses. Sci. Adv. 4, eaau1873 (2018).

38. Ings, R. M., McFadzean, J. A. \& Ormerod, W. E. The mode of action of metronidazole in Trichomonas vaginalis and other micro-organisms. Biochem. Pharmacol. 23, 1421-1429 (1974).

39. Davis, B., Glover, D. D. \& Larsen, B. Analysis of metronidazole penetration into vaginal fluid by reversed-phase high-performance liquid chromatography. Am. J. Obstet. Gynecol. 149, 802-803 (1984).

40. Simoes, J. A. et al. Human immunodeficiency virus type 1 stimulatory activity by Gardnerella vaginalis: relationship to biotypes and other pathogenic characteristics. J. Infect. Dis. 184, 22-27 (2001).
41. Brooks, J. P. et al. Changes in vaginal community state types reflect major shifts in the microbiome. Microb. Ecol. Health Dis. 28, 1303265 (2017).

42. Simoes, J. A., Aroutcheva, A. A., Shott, S. \& Faro, S. Effect of metronidazole on the growth of vaginal lactobacilli in vitro. Infect. Dis. Obstet. Gynecol. 9, 41-45 (2001).

43. De Backer, E. et al. Antibiotic susceptibility of Atopobium vaginae. BMC Infect. Dis. 6, 51 (2006).

44. Petrina, M. A. B., Cosentino, L. A., Rabe, L. K. \& Hillier, S. L. Susceptibility of bacterial vaginosis (BV)-associated bacteria to secnidazole compared to metronidazole, tinidazole and clindamycin. Anaerobe 47, 115-119 (2017).

45. Anukam, K. C. \& Reid, G. Effects of metronidazole on growth of Gardnerella vaginalis ATCC 14018, probiotic Lactobacillus rhamnosus GR-1 and vaginal isolate Lactobacillus plantarum KCA. Microb. Ecol. Health Dis. 20, 48-52 (2008).

46. Buffie, C. G. et al. Profound alterations of intestinal microbiota following a single dose of clindamycin results in sustained susceptibility to clostridium difficile-induced colitis. Infect. Immun. 80, 62-73 (2012).

47. France, M. T., Mendes-Soares, H. \& Forney, L. J. Genomic comparisons of Lactobacillus crispatus and Lactobacillus iners reveal potential ecological drivers of community composition in the vagina. Appl. Environ. Microbiol. 82 7063-7073 (2016)

48. O'Hanlon, D. E., Moench, T. R. \& Cone, R. A. Vaginal pH and microbicida lactic acid when lactobacilli dominate the microbiota. PLoS ONE 8, e80074 (2013).

49. Atassi, F., Pho Viet Ahn, D. L. \& Lievin-Le Moal, V. Diverse expression of antimicrobial activities against bacterial vaginosis and urinary tract infection pathogens by cervicovaginal microbiota strains of Lactobacillus gasseri and Lactobacillus crispatus. Front. Microbiol. 10, 2900 (2019).

50. Atassi, F., Brassart, D., Grob, P., Graf, F. \& Servin, A. L. Lactobacillus strain isolated from the vaginal microbiota of healthy women inhibit Prevotella bivia and Gardnerella vaginalis in coculture and cell culture. FEMS Immunol. Med. Microbiol. 48, 424-432 (2006).

51. Pybus, V. \& Onderdonk, A. B. Evidence for a commensal, symbiotic relationship between Gardnerella vaginalis and Prevotella bivia involving ammonia: potential significance for bacterial vaginosis. J. Infect. Dis. 175, 406-413 (1997).

52. Ravel, J. et al. Vaginal microbiome of reproductive-age women. Proc. Natl Acad. Sci. USA 108, 4680-4687 (2011).

53. Deng, Z.-L. et al. Metatranscriptome analysis of the vaginal microbiota reveals potential mechanisms for protection against metronidazole in bacterial vaginosis. mSphere 3, https://doi.org/10.1128/mSphereDirect.00262-18 (2018)

54. Brook, I. Inoculum effect. Rev. Infect. Dis. 11, 361-368 (1989).

55. Xiao, B. et al. Predictive value of the composition of the vaginal microbiota in bacterial vaginosis, a dynamic study to identify recurrence-related flora. Sci. Rep. 6, 26674 (2016).

56. Verwijs, M. C., Agaba, S. K., Darby, A. C. \& Wijgert, J. H. H. Mvande Impact of oral metronidazole treatment on the vaginal microbiota and correlates of treatment failure. Am. J. Obstet. Gynecol. 222, 157.e1-157.e13 (2020).

57. Allee, W. C. Principles of Animal Ecology (Saunders Co., 1949).

58. Goswami, M., Bhattacharyya, P. \& Tribedi, P. Allee effect: the story behind the stabilization or extinction of microbial ecosystem. Arch. Microbiol. 199, 185-190 (2017)

59. Stein, R. R. et al. Ecological modeling from time-series inference: insight into dynamics and stability of intestinal microbiota. PLoS Comput. Biol. 9, e1003388 (2013).

60. May, R. Stability and Complexity in Model Ecosystems (2001).

61. Medlock, G. L. et al. Inferring metabolic mechanisms of interaction within a defined gut microbiota. Cell Syst. 7, 245-257 (2018).

62. Momeni, B., Xie, L. \& Shou, W. Lotka-Volterra pairwise modeling fails to capture diverse pairwise microbial interactions. eLife 6, e25051 (2017).

63. Goldford, J. E. et al. Emergent simplicity in microbial community assembly. Science 361, 469-474 (2018).

64. McKendrick, A. G. \& Pai, M. K. XLV.-The rate of multiplication of micro organisms: a mathematical study. Proc. R. Soc. Edinb. 31, 649-653 (1912).

65. Marino, S., Hogue, I. B., Ray, C. J. \& Kirschner, D. E. A methodology for performing global uncertainty and sensitivity analysis in systems biology. $J$. Theor. Biol. 254, 178-196 (2008).

66. Narikawa, S. Distribution of metronidazole susceptibility factors in obligate anaerobes. J. Antimicrob. Chemother. 18, 565-574 (1986).

67. Guillén, H., Curiel, J. A., Landete, J. M., Muñoz, R. \& Herraiz, T. Characterization of a nitroreductase with selective nitroreduction properties in the food and intestinal lactic acid bacterium Lactobacillus plantarum WCFS1. J. Agric. Food Chem. 57, 10457-10465 (2009)

68. Altschul, S. F. \& Koonin, E. V. Iterated profile searches with PSI-BLAST-a tool for discovery in protein databases. Trends Biochem. Sci. 23, 444-447 (1998).

69. Chetwin, E. et al. Antimicrobial and inflammatory properties of South African clinical Lactobacillus isolates and vaginal probiotics. Sci. Rep. 9, 1917 (2019).

70. Juárez Tomás, M. S., Ocaña, V. S., Wiese, B. \& Nader-Macías, M. E. Growth and lactic acid production by vaginal Lactobacillus acidophilus CRL 1259, and 
inhibition of uropathogenic Escherichia coli. J. Med. Microbiol. 52, 1117-1124 (2003).

71. Castro, J., Rosca, A. S., Cools, P., Vaneechoutte, M. \& Cerca, N. Gardnerella vaginalis enhances Atopobium vaginae viability in an in vitro model. Front. Cell. Infect. Microbiol. 10, 83 (2020).

72. Gause, G. F. Experimental demonstration of Volterra's periodic oscillations in the numbers of animals. J. Exp. Biol. 12, 44-48 (1935).

73. Jackman, C. M., Deans, K. W., Forney, L. J. \& Lin, X. N. Microdroplet cocultivation and interaction characterization of human vaginal bacteria. Integr. Biol. 11, 69-78 (2019).

74. France, M. T., Mendes-Soares, H. \& Forney, L. J. Genomic comparisons of Lactobacillus crispatus and Lactobacillus iners reveal potential ecological drivers of community composition in the vagina. Appl. Environ. Microbiol. 82, 7063-7073 (2016).

75. CDC - STD Treatment. https://www.cdc.gov/std/treatment/default.htm (2020).

76. McKinnon, L. R. et al. The evolving facets of bacterial vaginosis: implications for HIV transmission. AIDS Res. Hum. Retroviruses 35, 219-228 (2019).

77. Lee, C. Y. et al. Quantitative modeling predicts mechanistic links between pre-treatment microbiome composition and metronidazole efficacy in bacterial vaginosis, chyylee/BV, https://doi.org/10.5281/zenodo.4121904 (2020).

\section{Acknowledgements}

This work was in part funded by startup funds from the University of Miami to N.R.K., funds from NIH/NIDDK grant RO1DK112254 to N.R.K., and NIH/NIAID grant R01AI138718 subcontract to N.R.K., and startup funds from the University of Michigan to K.B.A. The UMB-HMP study, J.R. and M.F. were supported by the National Institute for Allergy and Infectious Diseases of the National Institutes of Health under award numbers UH2AI083264 and R01NR015495. The CONRAD BV Study was funded by an intra-agency agreement between the Centers for Disease Control and Prevention (CDC), United States Aid and International Development (USAID) and CONRAD (GPO-A-0008-00005-00). The views expressed by the authors do not necessarily reflect those of the funding agency or CONRAD.

\section{Author contributions}

C.Y.L., R.K.C., M.M.L., N.R.K., and K.B.A. conceived and designed the study. C.Y.L. completed the computational analysis and analyzed the clinical data. R.K.C., N.R.K., and B.H. designed and conducted monoculture and co-culture kinetic experiments. A.G.,
M.F., and J.R. curated data from the UMB-HMP cohort. J.R. led the UMB-HMP study and data collection. A.T. and G.D. provided CONRAD BV protocol development, patien care, and data analysis. C.Y.L., R.K.C., K.B.A., and N.R.K. wrote the manuscript and all authors read and revised the manuscript.

\section{Competing interests}

J.R. is co-founder of LUCA Biologics, a biotechnology company focusing on translating microbiome research into live biotherapeutic drugs for women's health. All other authors declare no competing interests.

\section{Additional information}

Supplementary information is available for this paper at https://doi.org/10.1038/s41467 020-19880-w.

Correspondence and requests for materials should be addressed to N.R.K. or K.B.A.

Peer review information Nature Communications thanks the anonymous reviewers for their contribution to the peer review of this work. Peer reviewer reports are available.

Reprints and permission information is available at http://www.nature.com/reprints

Publisher's note Springer Nature remains neutral with regard to jurisdictional claims in published maps and institutional affiliations.

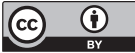

Open Access This article is licensed under a Creative Commons Attribution 4.0 International License, which permits use, sharing, adaptation, distribution and reproduction in any medium or format, as long as you give appropriate credit to the original author(s) and the source, provide a link to the Creative Commons license, and indicate if changes were made. The images or other third party material in this article are included in the article's Creative Commons license, unless indicated otherwise in a credit line to the material. If material is not included in the article's Creative Commons license and your intended use is not permitted by statutory regulation or exceeds the permitted use, you will need to obtain permission directly from the copyright holder. To view a copy of this license, visit http://creativecommons.org/ licenses/by/4.0/.

(C) The Author(s) 2020 